\title{
Numerical and analytical study of an atherosclerosis inflammatory disease model
}

\author{
A. Hidalgo - L. Tello - E. F. Toro
}

\begin{abstract}
We study a reaction-diffusion mathematical model for the evolution of atherosclerosis as an inflammation process by combining analytical tools with computer-intensive numerical calculations. The computational work involved the calculation of more than sixty thousand solutions of the full reaction-diffusion system and lead to the complete characterisation of the $\omega$-limit for every initial condition. Qualitative properties of the solution are rigorously proved, some of them hinted at by the numerical study.
\end{abstract}

Keywords Atherosclerosis - Finite volume formulation · Parabolic reactiondiffusion models - Asymptotic behaviour

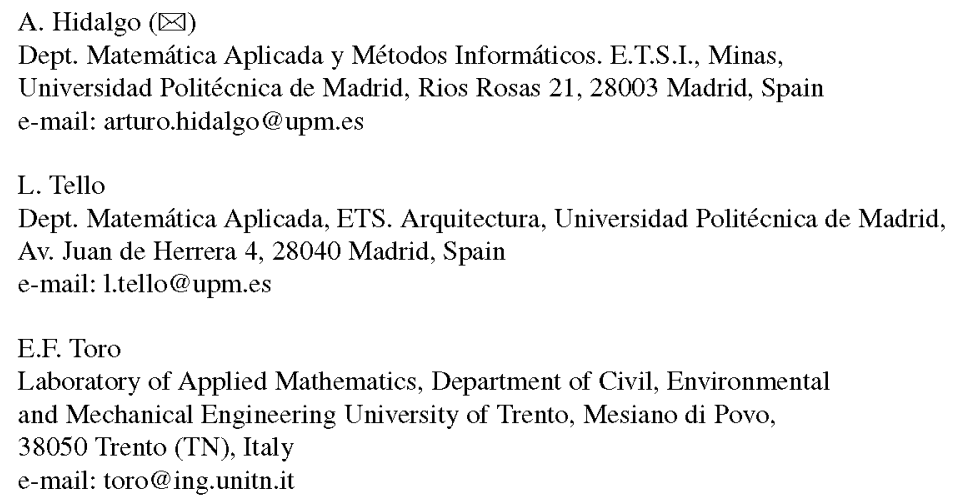




\section{Introduction}

This paper is concerned with the study of a reaction-diffusion mathematical model for the evolution of atherosclerosis as an inflammation process. Atherosclerosis is an anomalous condition affecting the wall of arterial vessels and is characterised by the formation of deposits of fatty materials, including cholesterol, and the local formation of plaques in the sub-endothelial intima of large arteries. Such plaques lead to atheromatous narrowing of the artery lumen, which in turn produces distal tissue ischaemia and thrombosis. Atherosclerosis, or atheroma, is not to be mistaken with arteriosclerosis. The latter refers generally to loss of elasticity of blood vessels as a consequence of a degenerative process due to ageing; arteriosclerosis is diffuse, rather than local; causes dilation of blood vessel, rather than narrowing, and raised pulse pressure, instead of distal ischaemia. Arteriosclerosis is common to all societies, while atherosclerosis is more specifically related to diet of westernised societies. For general background on physiological aspects of atherosclerosis see Levick (2010). Atherosclerosis is one of the main contributors to cardiovascular disease and is currently the leading cause of death and illness in some westernised countries (Libby 2002).

The initiation of atherosclerosis begins with the entry of low-density lipoproteins (LDLs) into the intima of the blood vessel, where they are oxidized (ox-LDLs). The body's immune system responds to the damage to the artery wall caused by oxidized LDL. The ox-LDL triggers the recruitment of the monocytes. The monocytes penetrate the intima and transform to macrophages which phagocyte the ox-LDL. Figure 1 illustrates the initiation of the atherosclerotic process. Ross and Glomset (1973) suggest that atherosclerosis is a response to injury to the vascular endothelium. The hypothesis that atherosclerosis is an inflammatory process is now accepted as a general concept amongst researchers in the field (Ross 1993). There are many causes of endothelial injury or disfunction. These include hyperlipidemia, diabetes, hypertension, smoking, viruses, hyperhomocystinemia, ischemia/reperfusion, etc. (Schachter 1997). Endothelial dysfunction leads to increase the permeability of the vessel walls. The macrophages phagocytose ox-LDL, but this eventually transforms them into foam cells (lipid-ladden cells) which in turn have to be removed by the immune system. At

Fig. 1 Schematic description of the beginning of the atherosclerosis process

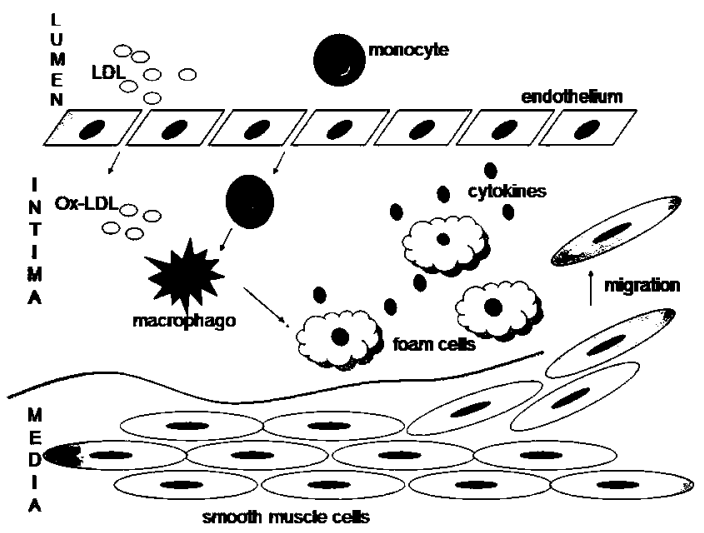


the same time they set up a chronic inflammatory reaction (auto-amplification phenomenon): they secrete pro-inflammatory cytokines (e.g. TNF- $\alpha, \mathrm{IL}-1$ ) which increase endothelial cells activation, promote the recruitment of new monocytes and support the production of new pro-inflammatory cytokines. This auto-amplification phenomenon is compensated by an anti-inflammatory phenomenon mediated by the antiinflammatory cytokines (e.g. IL-10), which inhibit the production of pro-inflammatory cytokines (biochemical anti-inflammation). Next, the inflammation process involves the proliferation and the migration of smooth muscle cells to create a fibrous cap over the lipid deposit which isolates this deposit centre from the blood stream (mechanical anti-inflammation).

Mathematical models are increasingly becoming an accepted methodology for studying and understanding complex phenomena in biology and medicine (Murray 2002; Cantrell and Cosner 2003). Concerning inflammatory response, in particular, different types of mathematical models can be found in the literature, see for example Chow et al. (2005); Herald (2010) and the references therein. More specifically, concerning mathematical models for atherosclerosis there are several approaches in the literature, depending on the degree of complexity of the models. For example, Calvez et al. (2010) study a very sophisticated model accounting for the coupling of the haemodynamics and mass transfer with a simple lesion growth model relying on the biomolecular process that takes place in the intima. The model involves fluidstructure interaction and non-linear systems of partial differential equations. Khatib et al. (2007) have proposed one and two-dimensional reaction-diffusion models to describe the response in the intima of the artery vessel. Related works are Cobbold et al. (2002); Li et al. (2006) and Ougrinovskaia et al. (2010).

In this paper we study numerically and analytically a reaction-diffusion model put forward by Khatib et al. (2009). The aim is to contribute to a more detailed understanding of the dynamics of the one-dimensional atherosclerosis mathematical model by combining numerical and analytical tools. In Sect. 2 we describe the mathematical model. In Sect. 3 we develop a sophisticated numerical method to compute accurate numerical solutions. The numerical methodology is based on a high-order non-linear fully discrete finite volume formulation of the ADER type (Toro et al. 2001). For a general introduction and references see Chaps. 19 and 20 of Toro (2009). In Sect. 4 numerical results are obtained for the atherosclerosis model. Concerning the asymptotic behaviour of the evolution solution, the characterisation of the initial data as a function of its $\omega$-limit is studied numerically in this section. This task is computer intensive, as for each of the three cases studied we need to solve more than twenty thousand times a reaction-diffusion system to high accuracy. Some of computational findings are then proved rigorously in the subsequent section. In the cases where one stationary solution represents disease and another one represents the healthy stationary state, the numerical method predicts if the initial lesion in a (perhaps very small) area of the artery wall can evolve to the healthy equilibrium or a disease equilibrium.

The numerical results obtained in this paper are qualitatively related to experimental observation reported in the literature; see for example Poston and Poston (2007); Ikeda and Torii (2005); Ye et al. (2011); Ibragimov et al. (2005). Some conclusions on this qualitative verification are given in Sect. 4.5. 
In Sect. 5 we carry out an analytical study of some qualitative, new, properties of the solution of the full reaction-diffusion model. Some of these properties were first discovered in the systematic computational study of the previous section.

\section{The mathematical model}

Here we consider the parabolic, one-dimensional reaction-difussion system proposed by Khatib et al. (2007). The governing equations are

$$
\begin{aligned}
\frac{\partial M}{\partial t} & =d_{1} \frac{\partial^{2} M}{\partial x^{2}}+f_{1}(A)-\lambda_{1} M, \quad x \in(0, L), t>0 \\
\frac{\partial A}{\partial t} & =d_{2} \frac{\partial^{2} A}{\partial x^{2}}+f_{2}(A) M-\lambda_{2} A, \quad x \in(0, L), t>0 \\
\frac{\partial M}{\partial x} & =0, \quad \frac{\partial A}{\partial x}=0 \quad \text { on } x=0 \text { and } x=L \\
M(x, 0) & =M_{0}(x), \quad A(x, 0)=A_{0}(x) .
\end{aligned}
$$

Here $M(x, t) \equiv$ density of immune cells (monocytes, macrophages); $A(x, t) \equiv$ density of cytokines secreted by immune cells; $t$ is the time; $x$ is the length along wall of the artery. The parameters $d_{1}$ and $d_{2}$ represent the diffusivity of the immune cells and cytokines, respectively. The positive parameters $\lambda_{1}$ and $\lambda_{2}$ represent the rate of degradation of the immune cells and the cytokines, respectively. The source term in the first equation, $f_{1}(A)$, is the recruitment of the immune cells from the blood stream, which is defined by

$$
f_{1}(A)=\frac{\alpha_{1}+\beta_{1} A}{1+\frac{A}{\tau_{1}}},
$$

with $\alpha_{1}=f_{1}(0)$ representing the beginning of the inflammation: the recruitment of monocytes due to the presence of ox-LDLs; $\beta_{1}$ is the auto-amplification of the recruitment of monocytes due to the inflammatory cytokines secreted by the monocytes; $\tau_{1}$ is the characteristic time for the fibrous cap formation; $1+\frac{A}{\tau_{1}}$ is the mechanical saturation of the recruitment of $\mathrm{M}$. The cytokine production rate is given by

$$
f_{2}(A)=\frac{\alpha_{2} A}{1+\frac{A}{\tau_{2}}},
$$

with $\alpha_{2} A$ representing the secretion of pro-inflammatory cytokines promoted by the pro-inflammatory cytokines themselves. $1+\frac{A}{\tau_{2}}$ is the inhibition of the proinflammatory cytokines secretion mediated by the anti-inflammatory cytokines. $\tau_{2}$ is the necessary time for this inhibition to act. All the parameters of the model, $\lambda_{1}, \lambda_{2}, d_{1}, d_{2}, \tau_{1}, \tau_{2}, \alpha_{1}, \alpha_{2}, \beta_{1}$, are positive.

Consequently $f_{2}$ is an increasing and bounded function; $f_{2}$ is an increasing function with a saturation effect due to the limited transfer rate of monocytes across the vessel wall. It is assumed 


$$
\tau_{1}>\frac{\alpha_{1}}{\beta_{1}}
$$

This hypothesis guarantees that $f_{1}$ is increasing, also bounded.

Although the model does not consider the thickness of the intima, it shows some essential features of atherosclerosis development. One characteristic of the model is the freedom to choose the initial data. This may indicate that the artery wall has an initial lesion in a particular small area and the model shows how it evolves along the artery wall. We could not analyze this fact through a model consisting of ordinary differential equations (ODEs).

Previous results about this model can be found in Khatib et al. (2009) where the authors obtain the constant stationary solutions for different sets of parameters for the model (1)-(4). They prove the existence of traveling waves connecting two constant steady states. Depending on the parameters of the model three cases can be distinguished in the kinetic system, an ODE system:

$$
\begin{aligned}
\frac{d M}{d t} & =f_{1}(A)-\lambda_{1} M \quad t \in(0,+\infty), \\
\frac{d A}{d t} & =f_{2}(A) M-\lambda_{2} A \quad t \in(0,+\infty), \\
M(0) & =M_{0}, \quad A(0)=A_{0} .
\end{aligned}
$$

The kinetic system has at least the equilibrium point $E_{0}=\left(\frac{\alpha_{1}}{\lambda_{1}}, 0\right)$. Khatib et al. (2007) have defined the following cases depending on the values of the parameters:

- Case I. If $\frac{\lambda_{2}}{\alpha_{2}}<\frac{\alpha_{1}}{\lambda_{1}}$ the ODEs system has one positive stable equilibrium point (monostable case).

- Case II. If $\frac{\alpha_{1}}{\lambda_{1}}<\frac{\lambda_{2}}{\alpha_{2}}<\frac{\beta_{1} \tau_{1}}{\lambda_{1}}$ and $0<\frac{\lambda_{2}}{\alpha_{2} \tau_{2}}<\left(\sqrt{\frac{\beta_{1}}{\lambda_{1}}-\frac{\alpha_{1}}{\tau_{1} \lambda_{1}}}-\sqrt{\frac{\lambda_{2}}{\alpha_{2} \tau_{1}}-\frac{\alpha_{1}}{\tau_{1} \lambda_{1}}}\right)^{2}$ then the ODEs system has two stable equilibria $E_{0}$ and $E_{r}$ and one unstable equilibrium (bistable case).

- Case III. If $\frac{\alpha_{1}}{\lambda_{1}}<\frac{\lambda_{2}}{\alpha_{2}}<\frac{\beta_{1} \tau_{1}}{\lambda_{1}}$ and $0<\frac{\lambda_{2}}{\alpha_{2} \tau_{2}}>\left(\sqrt{\frac{\beta_{1}}{\lambda_{1}}-\frac{\alpha_{1}}{\tau_{1} \lambda_{1}}}-\sqrt{\frac{\lambda_{2}}{\alpha_{2} \tau_{1}}-\frac{\alpha_{1}}{\tau_{1} \lambda_{1}}}\right)^{2}$ then the ODEs system has no positive equilibria. $E_{0}$ is stable.

We notice that every solution of (8)-(10) is a solution of (1)-(4) with constant initial datum. Moreover, every stationary solution of (8)-(10) is a (constant) stationary solution of (1)-(4), that is, it is a solution of the stationary problem:

$$
\begin{aligned}
& -d_{1} \frac{d^{2} M}{d x^{2}}+\lambda_{1} M=f_{1}(A) \\
& -d_{2} \frac{d^{2} A}{d x^{2}}+\lambda_{2} A=f_{2}(A) M \\
& \frac{d M}{d x}=0, \quad \frac{d A}{d x}=0 \quad \text { on } x=0 \text { and } x=L .
\end{aligned}
$$

In the next section we deal with the numerical approximation of the reactiondiffusion system (1)-(4). 


\section{Numerical approximation}

A substantial part of this paper consists of a detailed numerical study of reactiondiffusion system (1)-(4). To this end we develop a sophisticated numerical approximation method that ensures accurate and reliable results and whose use is completely under our control. The original program is available from the authors on request. We adopt the ADER approach in a finite volume framework. The resulting numerical approximation is non-linear and of arbitrary order of accuracy in space and time. The accuracy of the implemented scheme is assessed by studying convergence rates of the scheme.

\subsection{The finite volume framework}

We rewrite the system (1)-(4) as

$$
\frac{\partial \mathbf{Q}}{\partial t}=\mathbf{D} \frac{\partial^{2} \mathbf{Q}}{\partial x^{2}}+\mathbf{R}(\mathbf{Q})
$$

where

$$
\begin{aligned}
& \mathbf{Q}=(M, A)^{T}, \mathbf{D}=\left(\begin{array}{ll}
d_{1} & 0 \\
0 & d_{2}
\end{array}\right), \\
& \mathbf{R}=\left(f_{1}(A)-\lambda_{1} M, f_{2}(A) M-\lambda_{2} A\right)^{T} .
\end{aligned}
$$

Let us consider the control volume $V=\left[x_{i-\frac{1}{2}}, x_{i+\frac{1}{2}}\right] \times\left[t^{n}, t^{n+1}\right]$ in the $x-t$ half-plane, of dimensions

$$
\Delta x=x_{i+\frac{1}{2}}-x_{i-\frac{1}{2}}, \quad \Delta t=t^{n+1}-t^{n} .
$$

Now integrate exactly system (14) in space and time in $V$, giving the exact relation

$$
\mathbf{Q}_{i}^{n+1}=\mathbf{Q}_{i}^{n}+\frac{\Delta t}{\Delta x}\left(\mathbf{G}_{i+\frac{1}{2}}-\mathbf{G}_{i-\frac{1}{2}}\right)+\Delta t \mathbf{R}_{i},
$$

where

$$
\mathbf{Q}_{i}^{n}=\frac{1}{\Delta x} \int_{x_{i-\frac{1}{2}}}^{x_{i+\frac{1}{2}}} \mathbf{Q}\left(x, t^{n}\right) d x
$$

is the spatial integral average of $\mathbf{Q}$ at time $t^{n}$, called the cell average;

$$
\mathbf{G}_{i+\frac{1}{2}}=\frac{1}{\Delta t} \mathbf{D} \int_{t^{n}}^{t^{n+1}} \frac{\partial \mathbf{Q}}{\partial x}\left(x_{i+\frac{1}{2}}, t\right) d t
$$


is the temporal integral average of the spatial gradient of the solution at the interface $x=x_{i+1 / 2}$, while

$$
\mathbf{R}_{i}=\frac{1}{\Delta x \Delta t} \int_{t^{n}}^{t^{n+1}} \int_{x_{i-\frac{1}{2}}}^{x_{i+\frac{1}{2}}} \mathbf{R}(\mathbf{Q}(x, t)) d x d t
$$

is the space-time integral in $V$ of the complete source term.

A finite volume scheme of the form (17) results from approximating the appropriate integrals and denoting each term $\mathbf{T}$ by $\tilde{\mathbf{T}}$. Dropping the tildes we then interpret (17) as a numerical method. The numerical scheme is determined once a numerical flux $\mathbf{G}_{i+\frac{1}{2}}$ and a numerical source $\mathbf{R}_{i}$ are specified. Then scheme (17) allows the updating of the approximate cell average $\mathbf{Q}_{i}^{n}$ to the new approximate cell average $\mathbf{Q}_{i}^{n+1}$.

Here $\Delta x$ is the cell width, $x_{i-\frac{1}{2}}$ and $x_{i+\frac{1}{2}}$ are the cell interfaces; $x_{i}=\frac{1}{2}\left(x_{i-\frac{1}{2}}+x_{i+\frac{1}{2}}\right)$ is the cell centre and $\Delta t$ is the time step. In the next section we specify one methodology to determine the finite volume scheme.

\subsection{The ADER approach}

In this work we choose the ADER methodology, first introduced in the context of hyperbolic equations by Toro et al. (2001) and extensively used for several applications governed by hyperbolic equations [(see for instance Titarev and Toro $(2004,2005)]$. Recently, this methodology has been extended to reaction-diffusion equations (Toro and Hidalgo 2009; Gassner et al. 2007; Hidalgo and Dumbser 2011; Dumbser 2010).

First we describe the way to compute the numerical flux. It is convenient to interpret (19)-(20) in local coordinates, $(\xi, \tau)$, with $\xi$ representing $x-x_{i+\frac{1}{2}}$ and $\tau$ representing $t-t^{n}$, so that the interface $x_{i+1 / 2}$ is located at 0 and $t^{n}$ corresponds to 0 . Then the numerical flux at interface $x_{i+\frac{1}{2}}$ is expressed as

$$
\mathbf{G}_{i+\frac{1}{2}}=\frac{1}{\Delta t} \mathbf{D} \int_{0}^{\Delta t} \frac{\partial \mathbf{Q}}{\partial x}(0, \tau) d \tau
$$

and our aim is to calculate this to high accuracy in time. The procedure to achieve this contains two ingredients: (1) a high-order non-linear spatial reconstruction of the gradient of the solution in each cell and (2) the solution of the generalized (or high-order) Riemann problem at the interface of each cell. First we deal with the reconstruction problem. The usual way to proceed is to obtain reconstruction polynomials for each control volume which allow us to get approximate values and approximate derivatives of the unknown, $\mathbf{Q}(x, t)$, wherever they are needed. To circumvent Godunov's theorem (Godunov 1959) — which states that linear schemes cannot be monotone and of accuracy greater than one-we need to construct non-linear schemes even if applied to linear equations. To fulfill non-linearity we use a nonlinear reconstruction scheme, 
such as Essentially Non Oscillatory (ENO) or Weighted ENO (WENO) procedures. In this work we have used WENO reconstruction, which is profusely described in many references, see for instance Balsara and Shu (2000), Jiang and Shu (1996), Titarev and Toro (2004) and Hidalgo and Dumbser (2011). We notice that, since we are obtaining the numerical solution of a system of PDEs, we have to use vectors of polynomials. Therefore, in the rest of the paper, when referring to polynomials we actually mean vectors of polynomials.

Since we are working in one space dimension, when we want to obtain the reconstruction polynomial for the cell $i$ with an order of accuracy $r$ we have $r$ candidate stencils each one of them with $r$ cells. We can denote the $r$ stencils as $\left\{S_{i-r+1}, S_{i-r+2}\right.$, $\left.\cdots, S_{i}\right\},\left\{S_{i-r+2}, S_{i-r+3}, \cdots, S_{i+1}\right\}, \cdots,\left\{S_{i}, S_{i+1}, \cdots, S_{i+r-1}\right\}$. For each one of those stencils we can consider one $(r-1)-t h$ degree interpolating polynomial. Therefore we shall have $r$ polynomials: $\mathbf{P}_{l}(x), \quad l=0, \cdots, r-1$, each one of them of degree $r-1$. The reconstruction polynomial for cell $i$ is then obtained as a convex combination of the $r$ polynomials $\mathbf{P}_{l}$ taken with positive nonlinear weights. These weights are computed using the formulae

$$
\omega_{j}=\frac{\alpha_{j}}{\sum_{k=0}^{r-1} \alpha_{k}} \quad \text { where } \quad \alpha_{j}=\frac{d_{j}}{\left(\varepsilon+\beta_{j}\right)^{p}}, \quad(0 \leq j \leq r-1),
$$

where we use $p=2$ and $\varepsilon=10^{-24}$ which is introduced to avoid division by zero. In equation (22) $d_{j}$ are the linear weights while $\beta_{j}$ are the so-called smoothness indicators which are obtained from

$$
\beta_{k}=\sum_{m=0}^{r-1} \int_{x_{i-\frac{1}{2}}}^{x_{i+\frac{1}{2}}} \frac{d^{m}}{d x^{m}}\left(P_{k}(x)\right)^{2} \Delta x^{2 m-1} d x, \quad(0 \leq k \leq r-1)
$$

The linear weights, $d_{j}$, used in this work are based on those proposed by Dumbser et al. (2008) in which a very large weight is assigned to the central stencil and a very small linear weight is assigned to the biased ones. We remark that the reconstruction polynomials must be conservative, in the sense that the integral average of the polynomial must be equal to the integral average of the solution within each cell in the stencil

$$
\frac{1}{\Delta x_{k}} \int_{S_{k}} \mathbf{P}_{l}(x) d x=\mathbf{Q}_{k}, \quad 0 \leq(k, l) \leq r-1,
$$

where $S_{k}$ is each one of the $r$ cells in the stencil and $\Delta x_{k}$ is the cell width of cell $k$.

We now deal with the generalized Riemann problem to complete the computation of the numerical flux. Let us denote by $\mathbf{P}_{i}(x)$ and $\mathbf{P}_{i+1}(x)$ the vectors of polynomials for control volumes $i$ and $i+1$ respectively. To find the spatial gradient of the solution at the interface we solve following generalized Riemann problem of order $k$ 


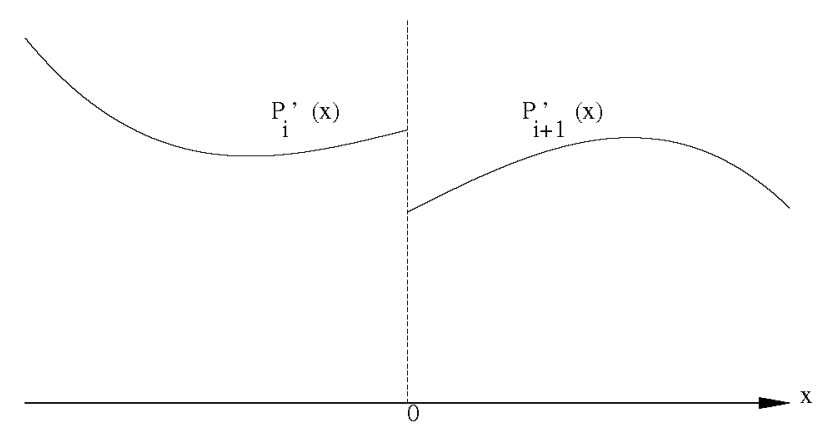

Fig. 2 Sketch of the initial condition for the generalized Riemann problem (25)

$$
\begin{gathered}
\frac{\partial}{\partial t}\left(\frac{\partial}{\partial x} \mathbf{Q}(x, t)\right)=\mathbf{D} \frac{\partial^{2}}{\partial x^{2}}\left(\frac{\partial}{\partial x} \mathbf{Q}(x, t)\right),-\infty<x<\infty, t>0, \\
\frac{\partial}{\partial x} \mathbf{Q}(x, 0)=\left\{\begin{array}{ll}
\frac{d}{d x} \mathbf{P}_{i}(x) & \text { if } x<0, \\
\frac{d}{d x} \mathbf{P}_{i+1}(x) & \text { if } x>0 .
\end{array}\right\}
\end{gathered}
$$

A general initial condition for (25) is depicted in Fig. 2, where we have used the notation $\mathbf{P}_{i}^{\prime}(x):=\frac{d}{d x} \mathbf{P}_{i}(x)$.

We note that it is easy to show that the spatial gradient of the solution $\mathbf{Q}(x, t)$ of the linear homogeneous second-order differential operator in (1)-(4) obeys identically the same operator, and thus we are justified in posing (25) to find the spatial gradient of $\mathbf{Q}(x, t)$ right at the interface as a function of time.

For the sake of simplicity we shall often use the notation $\mathbf{Q}^{(k)}(\tau):=\frac{\partial^{k}}{\partial x^{k}} \mathbf{Q}(0, \tau)$ when referring to spatial derivatives.

We seek the solution of (25) at $x=0, \mathbf{Q}^{(1)}(\tau)$, at a sufficiently small time $t=$ $\tau>0$. The solution is then smooth in time and we can express it via a Taylor series expansion (26)

$$
\mathbf{Q}^{(1)}(\tau)=\mathbf{Q}^{(1)}\left(0_{+}\right)+\sum_{k=1}^{K} \frac{\tau^{k}}{k !} \frac{\partial^{k}}{\partial t^{k}} \partial_{x} \mathbf{Q}\left(0,0_{+}\right)+\mathcal{O}\left(\tau^{K+1}\right)
$$

The leading term in the expansion (26), $\mathbf{Q}^{(1)}\left(0_{+}\right)$, is computed solving the following classical Riemann problem

$$
\begin{aligned}
& \frac{\partial}{\partial t}\left(\frac{\partial}{\partial x} \mathbf{Q}(x, t)\right)=\mathbf{D} \frac{\partial^{2}}{\partial x^{2}}\left(\frac{\partial}{\partial x} \mathbf{Q}(x, t)\right), \quad-\infty<x<\infty, \quad t>0, \\
& \frac{\partial}{\partial x} \mathbf{Q}(x, 0)= \begin{cases}\frac{d}{d x} \mathbf{P}_{i}(0) & \text { if } x \rightarrow 0_{-}, \\
\frac{d}{d x} \mathbf{P}_{i+1}(0) & \text { if } x \rightarrow 0_{+}\end{cases}
\end{aligned}
$$


The solution of (27) verifies

$$
\frac{\partial}{\partial x} \mathbf{Q}\left(0,0_{+}\right)=\frac{1}{2}\left(\frac{d}{d x} \mathbf{P}_{i+1}(0)+\frac{d}{d x} \mathbf{P}_{i}(0)\right) .
$$

Regarding the high order terms the time derivatives appearing in the expansion (26) can be transformed into spatial derivatives using the Cauchy-Kowalewski procedure, according to which we can write

$$
\frac{\partial^{k}}{\partial t^{k}}\left(\mathbf{Q}^{(1)}\left(0_{+}\right)\right)=H^{(k)}\left(\partial_{x} \mathbf{Q}\left(0,0_{+}\right), \partial_{x}^{(2)} \mathbf{Q}\left(0,0_{+}\right), \cdots, \partial_{x}^{(2 k+1)} \mathbf{Q}\left(0,0_{+}\right)\right)
$$

We note that to obtain the function $H^{(k)}$ we use the full PDE system, including the source terms. The spatial derivatives, which are the arguments of $H^{(k)}$, are obtained solving Riemann problems for spatial derivatives.

$$
\begin{aligned}
& \frac{\partial}{\partial t}\left(\frac{\partial^{l}}{\partial x^{l}} \mathbf{Q}(x, t)\right)=\mathbf{D} \frac{\partial^{2}}{\partial x^{2}}\left(\frac{\partial^{l}}{\partial x^{l}} \mathbf{Q}(x, t)\right),-\infty<x<\infty, t>0, \\
& \frac{\partial^{k}}{\partial x^{k}} \mathbf{Q}(x, 0)= \begin{cases}\frac{d^{l}}{d x^{l}} \mathbf{P}_{i}(0) & \text { if } x \rightarrow 0_{-}, \\
\frac{d^{l}}{d x^{l}} \mathbf{P}_{i+1}(0) & \text { if } x \rightarrow 0_{+} .\end{cases}
\end{aligned}
$$

The solution of (30) verifies

$$
\frac{\partial^{l}}{\partial x^{l}} \mathbf{Q}\left(0,0_{+}\right)=\frac{1}{2}\left(\frac{d^{l}}{d x^{l}} \mathbf{P}_{i+1}(0)+\frac{d^{l}}{d x^{l}} \mathbf{P}_{i}(0)\right)
$$

Once we have obtained the values of the leading and high order terms, they are used in the expansion (26) and, after integration, the numerical flux is obtained.

We also need to compute the integral of the source term given in (20) for which we use a Gaussian quadrature formula in order to perform the integration

$$
\int_{0}^{\Delta t} \int_{x_{i-\frac{1}{2}}}^{x_{i+\frac{1}{2}}} \mathbf{R}(\mathbf{Q}(x, t)) d x d t \approx \sum_{r=1}^{N} \omega_{r} \sum_{s=1}^{M} \mu_{s} \mathbf{R}\left(\mathbf{Q}\left(x_{r}, t_{s}\right)\right),
$$

where $N$ is the number of quadrature points (usually Gaussian ones) in $x$ while $M$ is the number of Gaussian points in $t$. The coefficients $\omega_{r}$ and $\mu_{s}$ are the weights in space and time respectively and $\left(x_{r}, t_{s}\right)$ are the integration points. In order to obtain $\mathbf{Q}\left(x_{r}, t_{s}\right)$ to be used in (32) we apply the following Taylor expansion 


$$
\begin{gathered}
\mathbf{Q}\left(x_{r}, \tau\right)=\mathbf{Q}\left(x_{r}, 0\right)+\sum_{k=1}^{K} \frac{\tau^{k}}{k !} \frac{\partial^{k}}{\partial t^{k}} \mathbf{Q}\left(x_{r}, 0\right)+\mathcal{O}\left(\tau^{K+1}\right), \\
(r=1, \cdots, N ; s=1 \cdots, M) .
\end{gathered}
$$

In an analogous manner to that used in the solution of the generalized Riemann problem to find the numerical flux, the time derivatives appearing in the expansion (33) are expressed as functions of space derivatives using the Cauchy-Kowalewski procedure. The leading term and high order terms are then calculated by means of spatial derivatives of the reconstruction polynomial inside the cell $i$.

\subsection{Numerical convergence test}

Here we verify the accuracy of our numerical scheme by studying empirical convergence rates. To this end we use an analogue of the atherosclerosis model (1)-(4), also of the reaction-diffusion type but with known analytical solution. The analogue, as every analogue should, has all the ingredients appearing in the atherosclerosis model, so it is suitable for, at least partially, validating the numerical method. The analogue is

$$
\begin{gathered}
\frac{\partial q(x, t)}{\partial t}-\frac{\partial}{\partial x}\left((1+q(x, t))^{2} \frac{\partial q(x, t)}{\partial x}\right)+0.5 q(x, t)=S(x, t) \\
\frac{\partial q}{\partial x}=0, \text { on } x=-10 \text { and } x=10 \\
q(x, 0)=\exp \left(-\frac{x^{2}}{4}\right)+\sin \left(\frac{\pi x}{20}\right)
\end{gathered}
$$

where $x \in \Omega=(-10,10), t \in(0,0.25)$. The forcing term is

$$
\begin{aligned}
S(x, t)=- & e^{\beta(x, t)}-2\left(e^{\beta(x, t)}+\sin \alpha(x)\right)\left(-\frac{x}{2} e^{\beta(x, t)}+\frac{\pi}{20} \cos \alpha(x)\right)^{2} \\
& -\left(1+\left(e^{\beta(x, t)}+\sin \alpha(x)\right)^{2}\right)\left(-\frac{1}{2} e^{\beta(x, t)}+\frac{x^{2}}{4} e^{\beta(x, t)}-\frac{\pi^{2}}{400} \sin \alpha(x)\right) \\
& +\frac{1}{4}\left(e^{\beta(x, t)}+\sin \alpha(x)\right)
\end{aligned}
$$

where $\beta(x, t)=-\left(\frac{x^{2}}{4}+t\right)$ and $\alpha(x)=\frac{\pi x}{20}$. The exact solution of (34)-(37) is

$$
q(x, t)=\exp \left(-\frac{x^{2}}{4}+t\right)+\sin \left(\frac{\pi x}{20}\right)
$$

We solve the auxiliary test problem (34)-(37) using 2nd and 3rd order ADER finite volume schemes for various meshes to be specified. To achieve 2 nd and 3 rd accuracy we require polynomial reconstruction of 3 rd and 5 th degrees, respectively. 


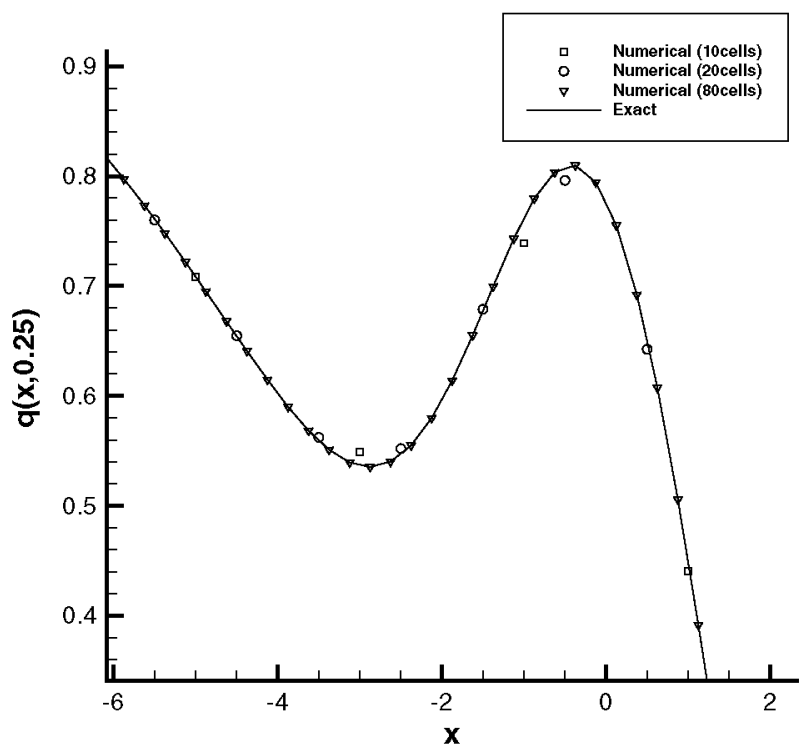

Fig. 3 Comparison of the numerical solution (symbols) with the exact solution (full line) of the auxiliary problem for three meshes

We first use the exact solution to compare it with the numerical solution, as depicted in Fig. 3, which shows such comparison of the numerical solution (shown in symbols) with the exact solution (shown by full line) of the auxiliary problem, for three meshes, at the fixed time $t=0.25$. Note that we display only part of the computational domain in Fig. 3. It is seen that the numerical method captures the solution very accurately, even for coarse meshes.

The second way we use the exact solution of the auxiliary problem is to carry out a convergence rate study to verify the theoretically expected accuracy of the schemes. Table 1 shows the errors measured in three usual norms and the corresponding convergence rates. It can be seen that the order of accuracy is larger than expected theoretically, in both cases. We remark that the meshes used are quite coarse, in order to avoid the influence of the machine precision in the computation of the convergence rates. The results obtained here suggest that the adopted ADER numerical approach is suitable to study the atherosclerosis model of interest in this work.

\section{Numerical study of the atherosclerosis model}

The aim of this section is twofold. First we utilise the numerical method developed in the previous section to solve the reaction-diffusion model (1)-(4) to study the effect of the size of the initial conditions in the time-evolved solution, for both the monostable and bistable cases. The second aim is to utilise the numerical tool to establish a relationship between classes of initial conditions and steady-state solutions 
Table 1 Convergence rates for auxiliary problem

\begin{tabular}{cllllll}
\hline Cells & $\|$ Error $\|_{1}$ & $\|$ Order $\|_{1}$ & $\|$ Error $\|_{2}$ & $\|$ Order $\|_{2}$ & $\|$ Error $\|_{\infty}$ & $\|$ Order $\|_{\infty}$ \\
\hline ADER-2 & & & & & \\
10 & $3.38 \times 10^{-3}$ & & $2.59 \times 10^{-2}$ & & $1.45 \times 10^{-2}$ & \\
20 & $3.43 \times 10^{-4}$ & 3.30 & $2.96 \times 10^{-3}$ & 3.13 & $2.35 \times 10^{-3}$ & 2.63 \\
40 & $1.54 \times 10^{-5}$ & 4.47 & $1.35 \times 10^{-4}$ & 4.46 & $8.46 \times 10^{-5}$ & 4.79 \\
80 & $8.90 \times 10^{-7}$ & 4.11 & $7.39 \times 10^{-6}$ & 4.19 & $5.94 \times 10^{-6}$ & 3.83 \\
ADER-3 & & & & & $7.19 \times 10^{-2}$ & \\
10 & $1.56 \times 10^{-2}$ & & $0.14 \times 10^{0}$ & & $7.07 \times 10^{-3}$ & 3.35 \\
20 & $8.06 \times 10^{-4}$ & 4.28 & $1.00 \times 10^{-3}$ & 3.82 & $6.69 \times 10^{-5}$ & 6.72 \\
40 & $9.09 \times 10^{-6}$ & 6.47 & $8.31 \times 10^{-4}$ & 6.91 & $1.15 \times 10^{-6}$ & 5.87 \\
80 & $2.06 \times 10^{-7}$ & 5.47 & $1.68 \times 10^{-6}$ & 5.63 & & \\
\hline
\end{tabular}

Output time $t=0.25$

of the full time-dependent model (1)-(4), and also to use the numerical findings as hints to rigorously prove some new results in Sect. 5 .

For all the calculations of this section, we have used the mesh $\Delta x=0.01$ and the calculation time step has been computed from the stability constraint $\Delta t=$ $\min \left(\kappa \Delta x^{2} / d_{1}, \kappa \Delta x^{2} / d_{2}\right)$. For the calculations of Sect. 4.1 we use $\kappa=0.45$ and for those of Sect. 4.2 we use $\kappa=0.01$.

\subsection{Effect of initial conditions}

For the purpose of studying the effect of the size of the initial conditions in the time-evolved solution we consider the following families of initial conditions $M_{0}(x), A_{0}(x)$, taken as perturbations of the stationary state $(\hat{M}, \hat{A})=(2,0)$, namely

$$
M_{0}(x)=\hat{M}+\epsilon \varphi(x), \quad A_{0}(x)=\hat{A}+\epsilon \varphi(x),
$$

where

$$
\varphi(x)=e^{-(20 x-10)^{2}}
$$

is the perturbation. As to the coefficient $\epsilon$, we have taken $\epsilon=0.2$ and $\epsilon=3.0$ for small and large perturbations respectively. For the bio-mechanical parameters we have adopted those in Table 2, taken from the literature, see Khatib et al. (2009, 2012), Edelstein-Keshet and Spiros (2002) and Chow et al. (2005). As to the diffusion coefficients, for the calculations of this section we take $d_{1}=10^{-2} \mathrm{~cm}^{2} / \mathrm{h}$ for the immune cells and $d_{2}=10^{-1} \mathrm{~cm}^{2} / h$ for the cytokines.

Computed results are shown in Figs. 4 and 5 for the bistable case and in Figs. 6 and 7 for the monostable case. Fig. 4 shows results for the bistable case, with initial condition consisting of a small perturbation of the healthy steady state. The top frame shows the 
Table 2 Parameters for the numerical simulation

\begin{tabular}{llllllllll}
\hline$L=1$ & $\alpha_{1}$ & $\alpha_{2}$ & $\beta_{1}$ & $\tau_{1}$ & $\tau_{2}$ & $\lambda_{1}$ & $\lambda_{2}$ & $d_{1}$ & $d_{2}$ \\
\hline bistable & 2 & 7 & 8 & 1 & 6.5 & 1 & 26 & $10^{-5}$ & $10^{-4}$ \\
monostable & 2 & 1 & 8 & 1 & $42 / 43$ & 1 & 1 & $10^{-5}$ & $10^{-4}$ \\
\hline
\end{tabular}
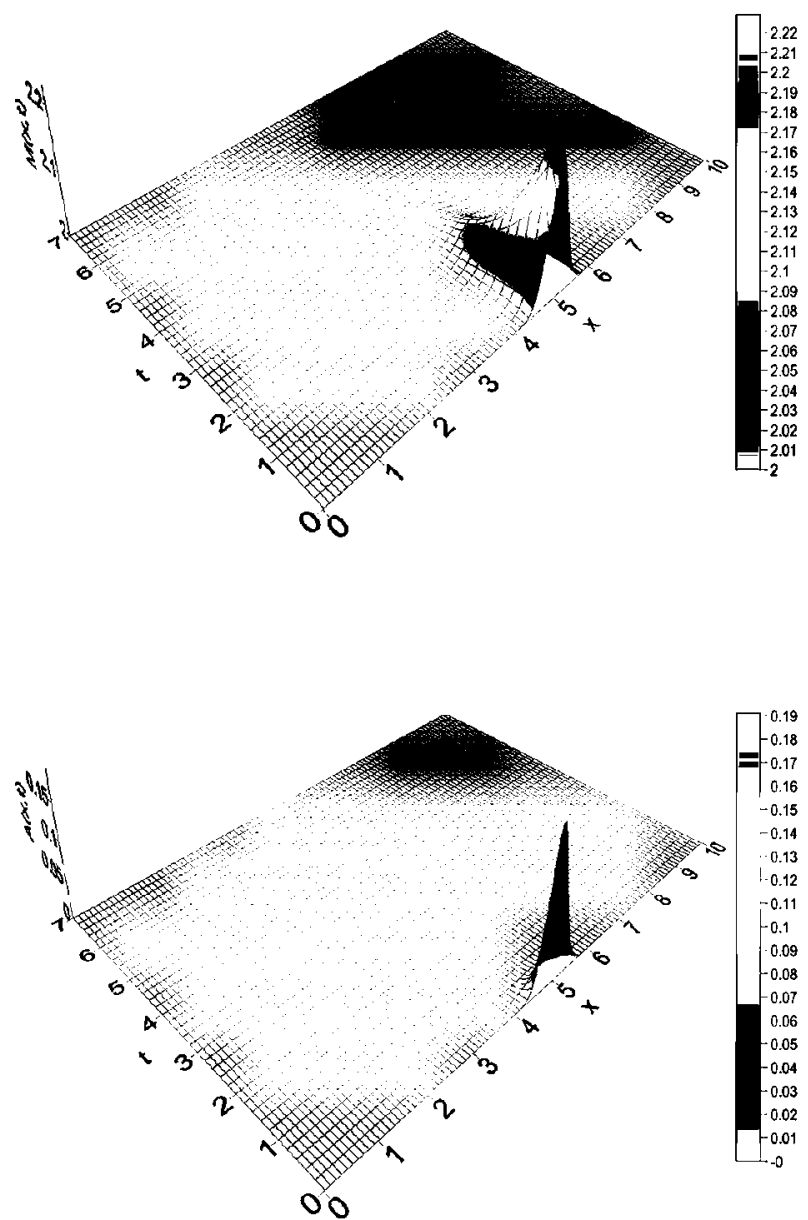

Fig. 4 Bistable case. Initial condition: small perturbation of healthy steady state. Top frame Density $\mathrm{M}(\mathrm{x}, \mathrm{t})$ of immune cells. Bottom frame Density $\mathrm{A}(\mathrm{x}, \mathrm{t})$ of cytokines

density $M(x, t)$ of the immune cells, while the bottom frame shows the density $A(x, t)$ of cytokines. The results tell us that these initial conditions lead to a healthy state, the initial inflammation dies out, to a steady state, as time evolves. Figure 5 shows results for the bistable case, with initial condition consisting of a large perturbation of the healthy steady state. The top frame shows the density $M(x, t)$ of the immune cells, while the bottom frame shows the density $A(x, t)$ of cytokines. The results tell us that 

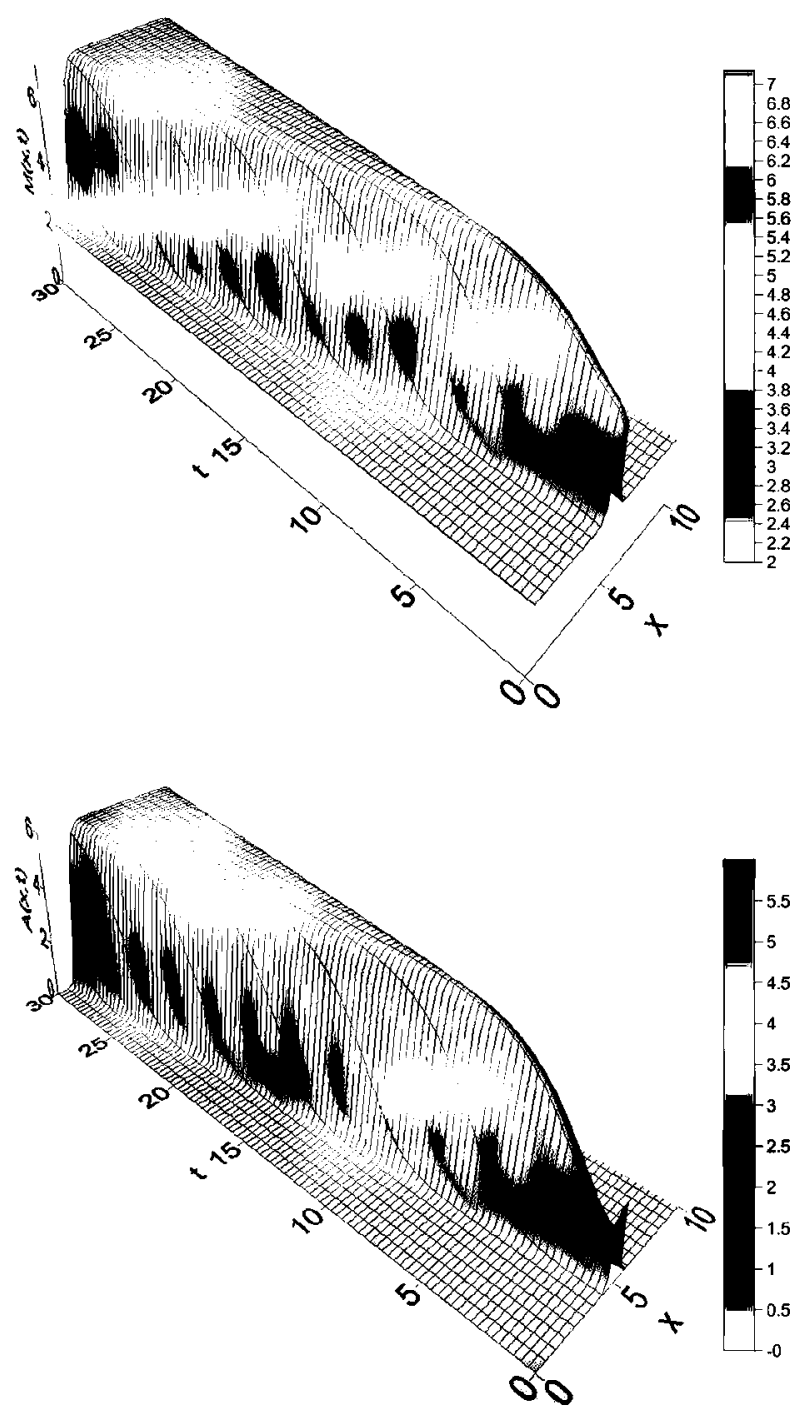

Fig. 5 Bistable case. Initial condition: large perturbation of healthy steady state. Top frame Density $\mathrm{M}$ (x,t) of immune cells. Bottom frame Density $\mathrm{A}(\mathrm{x}, \mathrm{t})$ of cytokines

these initial conditions lead to an inflammatory state, the initial inflammation grows to a steady state, as time evolves. Figure 6 shows results for the monostable case, with initial condition consisting of a small perturbation of the healthy steady state. The top frame shows the density $M(x, t)$ of the immune cells, while the bottom frame shows the density $A(x, t)$ of cytokines. The results tell us that these initial conditions lead to an inflammatory state, the initial inflammation grows to a steady state, as time evolves. Figure 7 shows results for the monostable case, with initial condition consisting of a large perturbation of the healthy steady state. The top frame shows the density $M(x, t)$ 

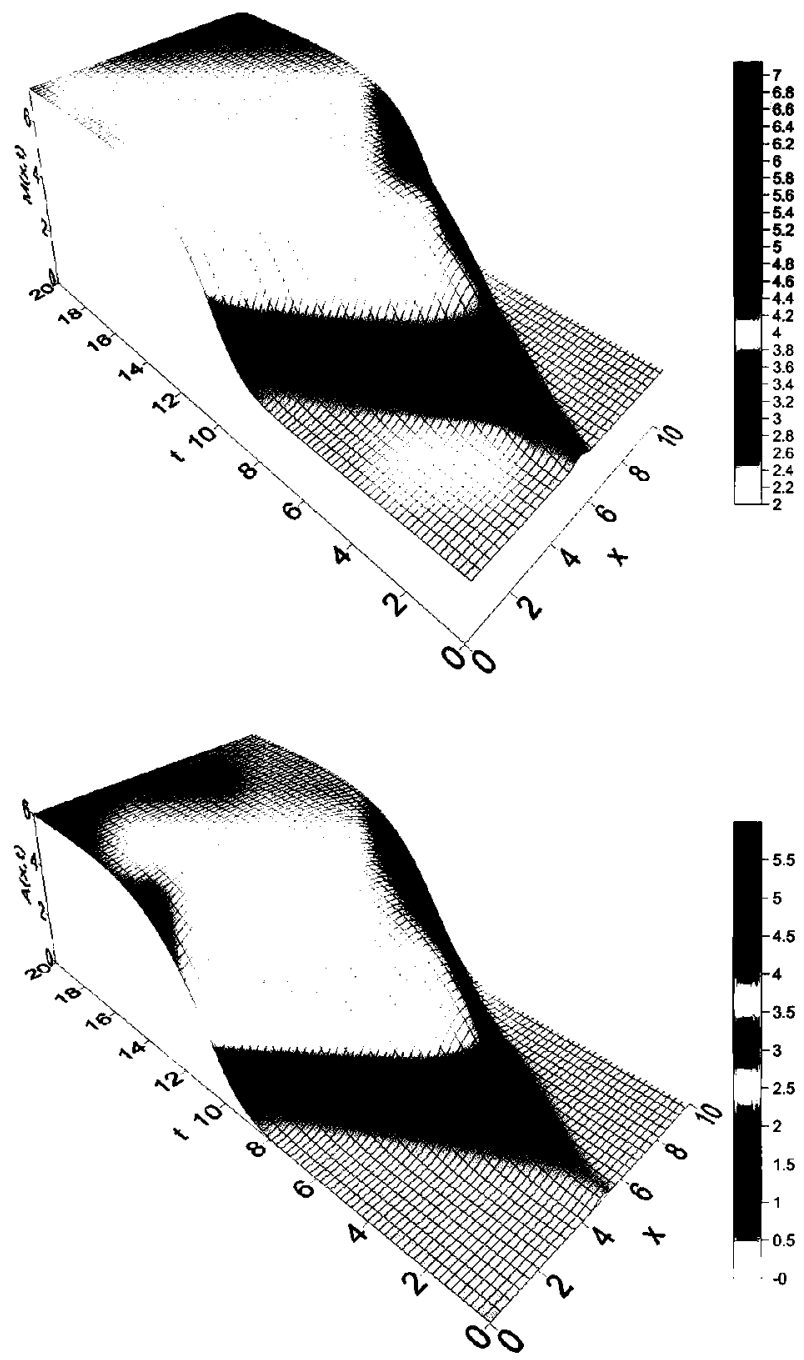

Fig. 6 Monostable case. Initial condition: small perturbation of healthy steady state. Top frame density $\mathrm{M}(\mathrm{x}, \mathrm{t})$ of immune cells. Bottom frame Density $\mathrm{A}(\mathrm{x}, \mathrm{t})$ of cytokines

of the immune cells, while the bottom frame shows the density $A(x, t)$ of cytokines. The results tell us that these initial conditions lead to an inflammatory state, the initial inflammation grows to a steady state, as time evolves.

The above results confirm those of Khatib et al. (2007), even though we have used different diffusion coefficients and a different numerical technique in our calculations.

\subsection{Characterisation of initial conditions in the phase plane}

Motivated by the previous numerical results we now perform an intensive computational task to carry out a numerical study of regions of attraction of station- 

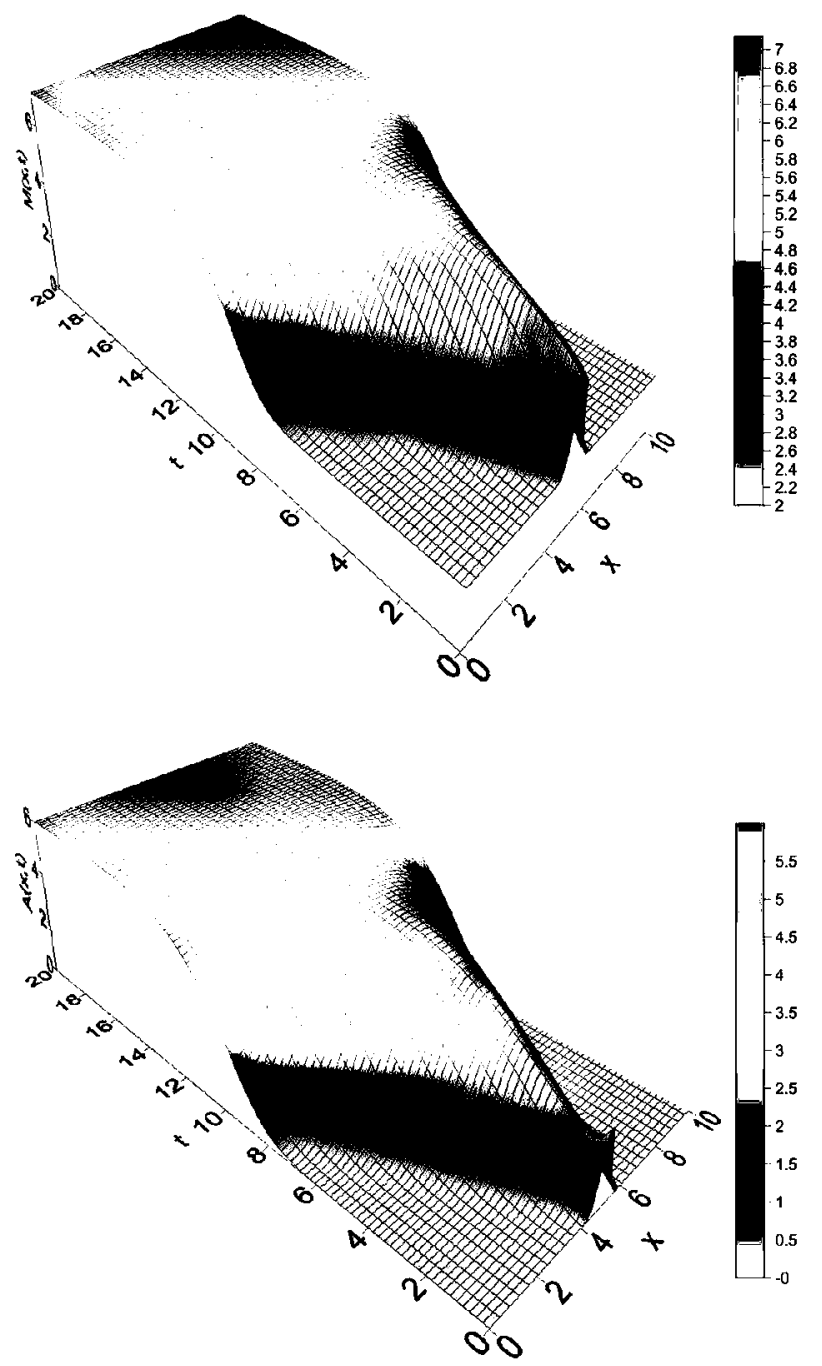

Fig. 7 Monostable case. Initial condition: large perturbation of healthy steady state. Top frame Density $\mathrm{M}(\mathrm{x}, \mathrm{t})$ of immune cells. Bottom frame Density $\mathrm{A}(\mathrm{x}, \mathrm{t})$ of cytokines

ary states of system (1)-(4). The results of the numerical study allow us to then prove rigorously some of the numerical findings. Since we know that for some choice of parameters the problem has more than one stable stationary state, the limit $\lim _{t \rightarrow+\infty}(M(x, t), A(x, t))$ depends on the initial condition. The computed steady state solutions of (1)-(4) described here coincide with the steady solutions theoretically obtained.

To compute steady-state solutions of (1)-(4) we apply the numerical scheme described in Sect. 3, to solve the time-dependent system (1)-(4) in a time-marching fashion according to Algorithm $S^{3}$ described below. This is done in order to characterise the initial data leading to a disease equilibrium or a healthy equilibrium. 
Obviously, we do not introduce any information in the numerical scheme as to the stationary solution; the time-marching procedure finds it automatically, as time limit of the time-dependent solution.

Now recall the definition of the $\omega$-limit set of a solution. Let $(M, A)$ be a solution of the parabolic problem (1)-(4), we define the $\omega$-limit set of $(M, A)$ by

$$
\begin{aligned}
\omega(M, A)=\{( & \left.M_{\infty}, A_{\infty}\right) \in H^{1}(\Omega) \times H^{1}(\Omega): \exists t_{n} \rightarrow+\infty \text { such that } \\
& \left.\left(M\left(t_{n}, \cdot\right), A\left(t_{n}, \cdot\right)\right) \rightarrow\left(M_{\infty}, A_{\infty}\right) \text { in } L^{2}(\Omega) \times L^{2}(\Omega)\right\} .
\end{aligned}
$$

Let us now anticipate a theoretical result proved in Sect. 5. Proposition 1 tells us that for initial data $\left(M_{0}(x), A_{0}(x)\right)$ verifying $\frac{\alpha_{1}}{\lambda_{1}} \leq M_{0}(x) \leq \frac{\beta_{1} \tau_{1}}{\lambda_{1}}$ and $0 \leq$ $A_{0}(x) \leq \frac{\beta_{1} \tau_{1} \alpha_{2} \tau_{2}}{\lambda_{1} \lambda_{2}}$, the corresponding evolutionary solutions of (1)-(4) also satisfy $\frac{\alpha_{1}}{\lambda_{1}} \leq M(x, t) \leq \frac{\beta_{1} \tau_{1}}{\lambda_{1}}$ and $0 \leq A(x, t) \leq \frac{\beta_{1} \tau_{1} \alpha_{2} \tau_{2}}{\lambda_{1} \lambda_{2}}$. According to Proposition 2 the stationary solutions also verify these bounds.

We remark here that in the rest of the paper, when we use $\omega$-limit of an initial datum, we mean the $\omega$-limit of the time-dependent solution of (1)-(4) with such initial datum.

For the computational problem we therefore define the region of interest as the rectangle

$$
\mathcal{R}=\left\{(A, M) \in \mathbb{R}^{2}: 0 \leq A \leq \frac{\beta_{1} \tau_{1} \alpha_{2} \tau_{2}}{\lambda_{1} \lambda_{2}}, \quad \frac{\alpha_{1}}{\lambda_{1}} \leq M \leq \frac{\beta_{1} \tau_{1}}{\lambda_{1}}\right\}
$$

We consider a very large number of initial data and compute their $\omega$-limit. We have designed three large families of initial data and have classified those initial data into two sets according to the computational results. The first set (second set) is formed by initial data whose $\omega$-limit is a healthy stationary state (disease stationary state). These two sets are identified with two distinct regions of the rectangle $\mathcal{R}$. Note that in the definition of $\mathcal{R}$ we have changed the order of the coordinates in order to display a clear graphical representation.

We define a family $\mathcal{M}$ composed of $m \times n$ small squares in the rectangle $\mathcal{R}$ and we denote by $\left(A_{j}, M_{i}\right)=\left(0+\left(j-\frac{1}{2}\right) k, \frac{\alpha_{1}}{\lambda_{1}}+\left(i-\frac{1}{2}\right) h\right)$ the centre of every square, for $i=1, \ldots, m ; j=1, \ldots, n$.

Next we identify every point $\left(A_{j}, M_{i}\right)$ with an initial datum $U_{i j}(x)=\left(M_{i}^{0}(x)\right.$, $\left.A_{j}^{0}(x)\right)$. We compute the $\omega$-limit of the solution of (1)-(4) with such initial datum and obtain a computed stationary solution. Then we classify that initial datum as an element of the attraction region of such stationary solution.

Every point $\left(A_{j}, M_{i}\right)$ in $\mathcal{R}$ is associated to a colour depending on the $\omega$-limit of $U_{i j}(x)$. This colour is also assigned to the full small square of which $\left(A_{j}, M_{i}\right)$ is the centre.

Let us now consider the bistable case and choose different families of initial data to show its evolution towards one stationary solution or the other (healthy or disease). We have considered a very large number of $96 \times 224=21,504$ initial data to get the results displayed in Figs. 9, 10 and 11. This is a very intensive computational problem as for each of the 21, 504 cases we have solved the reaction-diffusion system (1)-(4) to steady state according to the algorithm described in Sect. 4.4. 

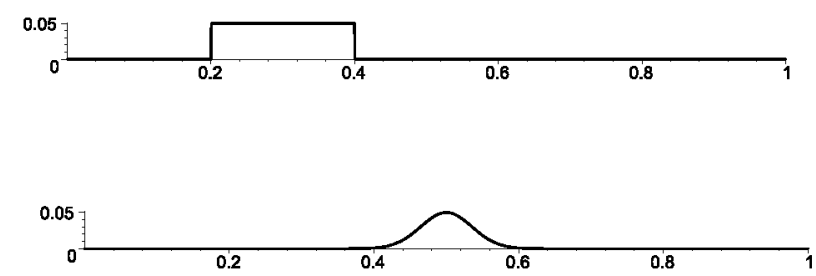

Fig. 8 Nonconstant initial datum type in Example 2 (initial perturbation). The bottom graph represents the nonconstant initial data type in Example 3 (Gaussian perturbation)

For the data of Table 2 the rectangle $\mathcal{R}$ in (39) becomes $\mathcal{R}=[0,14] \times[2,8]$.

The two stable (constant) stationary solutions are given by:

$$
M_{0}=\frac{\alpha_{1}}{\lambda_{1}}=2, \quad A_{0}=0
$$

and

$$
M_{r}:=\frac{f_{1}\left(A_{r}\right)}{\lambda_{1}}=\frac{50}{7} \sim 7.142857143, \quad A_{r}:=6 .
$$

The unstable stationary solution is given by

$$
M_{u n s}:=\frac{f_{1}\left(A_{u n s}\right)}{\lambda_{1}}=4, \quad A_{u n s}:=0.5
$$

Example 1 We start by considering constant initial data. We have approximated the associated evolution solution and we have estimated the limit at infinity. The considered family of initial data is given by

$$
M_{i}^{0}(x)=2+\left(i-\frac{1}{2}\right) h, \quad A_{j}^{0}(x)=\left(j-\frac{1}{2}\right) k
$$

where $i=1, \ldots, m ; j=1, \ldots, n, h=0.0625, k=0.0625$. Every initial datum $\left(M_{i}^{0}(x), A_{j}^{0}(x)\right)$ is identified with the centre $\left(A_{j}, M_{i}\right)$ of every square of $\mathcal{M}$. The classification of these initial data is displayed in Fig. 9.

Example 2 We consider nonconstant initial data. We build a new family of initial data by perturbing the initial data of Example 1 (represented in Fig. 8). Therefore we consider

$$
M_{i}^{0}(x)=2+\left(i-\frac{1}{2}\right) h+\epsilon \chi_{S}(x) \quad A_{j}^{0}(x)=0+\left(j-\frac{1}{2}\right) k+\epsilon \chi_{S}(x)
$$

where $i=1, \ldots, m ; j=1, \ldots, n, h=0.0625, k=0.0625, \epsilon=0.05$ and $\chi_{S}(x)$ is the characteristic function of the interval $S=(0.2,0.4)$. Every initial datum $\left(M_{i}^{0}(x), A_{j}^{0}(x)\right.$ is identified with the centre $\left(A_{j}, M_{i}\right)$ of every square. The classification of these initial data is displayed in Fig. 10. 


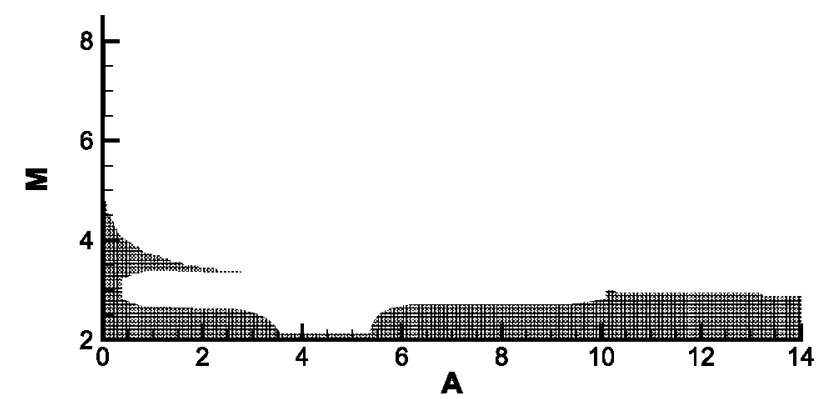

Fig. 9 Example 1. Constant initial data, plotted in the phase plane, where colors depend on the $\omega$-limit of the initial data. In every case the $\omega$-limit is one of the stationary solutions of the system

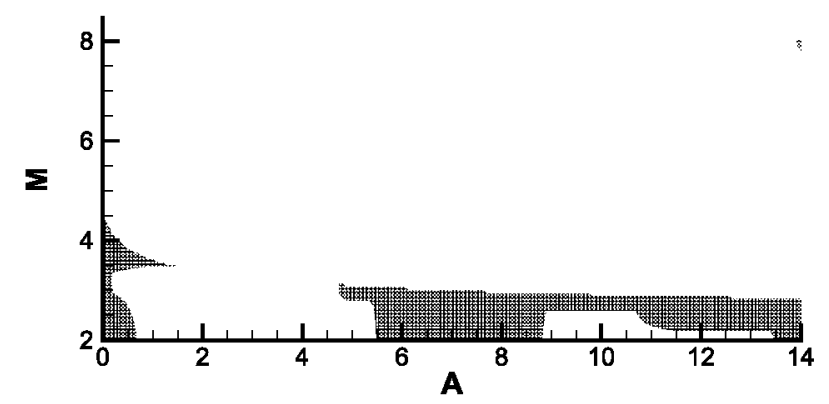

Fig. 10 Example 2. Small constant perturbation of initial data of Example 1, plotted in the phase plane, where colors depend on the $\omega$-limit of the initial data. In every case the $\omega$-limit is one of the stationary solutions of the system

Example 3 Now, we consider nonconstant initial data in the invariant rectangle. We build a new family of initial data by perturbing the initial data of Example 1 (represented in Fig. 8). So,

$$
M_{i}^{0}(x)=2+\left(i-\frac{1}{2}\right) h+\epsilon \phi(x), \quad A_{j}^{0}(x)=0+\left(j-\frac{1}{2}\right) k+\epsilon \phi(x)
$$

where $i=1, \ldots, m ; j=1, \ldots, n, h=0.0625, k=0.0625$ and $\phi(x)=$ $e^{-(20 x-10)^{2}}$ and $\epsilon=0.05$. Every initial datum $\left(M_{i}^{0}(x), A_{j}^{0}(x)\right.$ is identified with the centre $\left(A_{j}, M_{i}\right)$ of every square. The classification of these initial data is displayed in Fig. 11. The colour of every square with center $\left(A_{j}, M_{i}\right)$ indicates in which attraction region is the initial datum $U_{i j}(x)$ of this example.

\subsection{Discussion}

For a certain range of parameters the atherosclerosis model has at least two stationary states simulating the healthy state and the disease state.

We focus our attention in a rectangular region where we consider the initial data and the range of parameters corresponding to the bistable case. In Figs. 9, 10, 11 we can see 


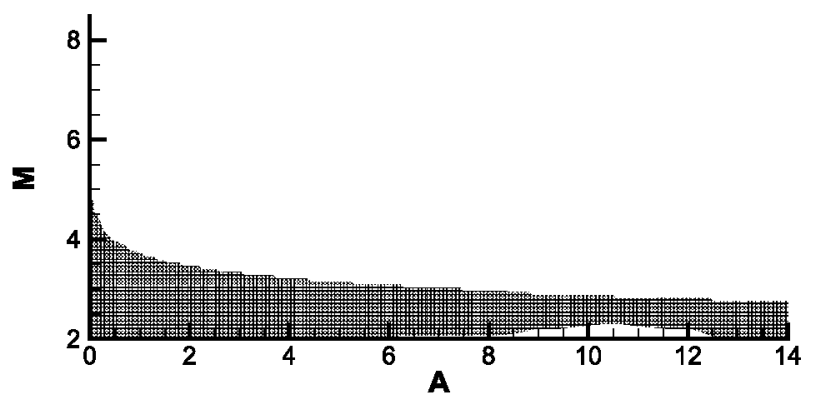

Fig. 11 Example 3. Small Gaussian perturbation of initial data of Example 1, plotted in the phase plane, where colors depend on the $\omega$-limit of the initial data. In every case the $\omega$-limit is one of the stationary solutions of the system

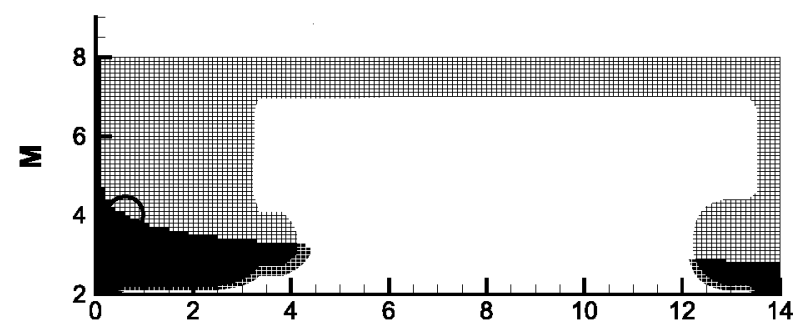

A

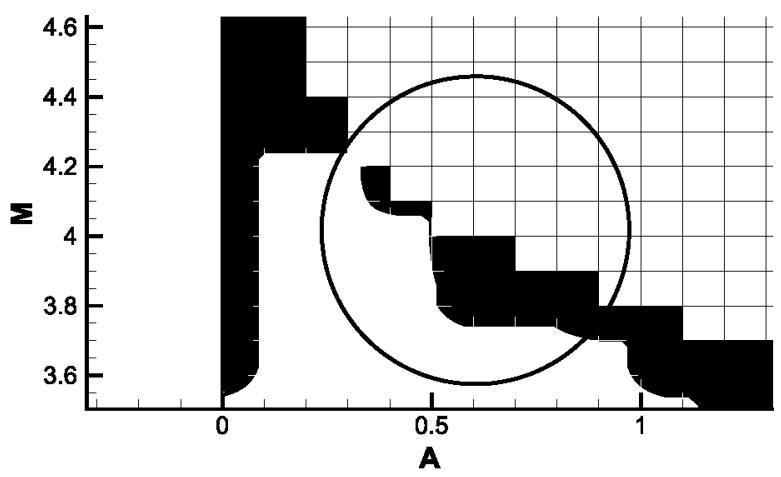

Fig. 12 Example 1 revisited. Top frame Constant initial data plotted in the phase plane where colors depend on the $\omega$-limit of them. In this particular case one of the ICs considered is precisely the unstable steady state solution, so its $\omega$-limit is the IC itself. Bottom frame Zoom of three-colour zone where the third colour appears at the position $(0.5,4.0)$ (color figure online)

the region of attraction of the stationary states. Each of these figures has two regions. The upper region represents the initial data in the chosen family of every example which evolve to the disease steady state. The lower region represents the initial data in the chosen family of every example which evolve to the healthy steady state.

The unstable stationary solution is only detected when it is a centre of a square of the mesh. Figure 12 depicts the situation in which the unstable steady state $(0.5,4)$ is an initial condition. In this case, a third color appears as the numerical solution remains with the same value as time is evolving, since it is a steady state solution. We 


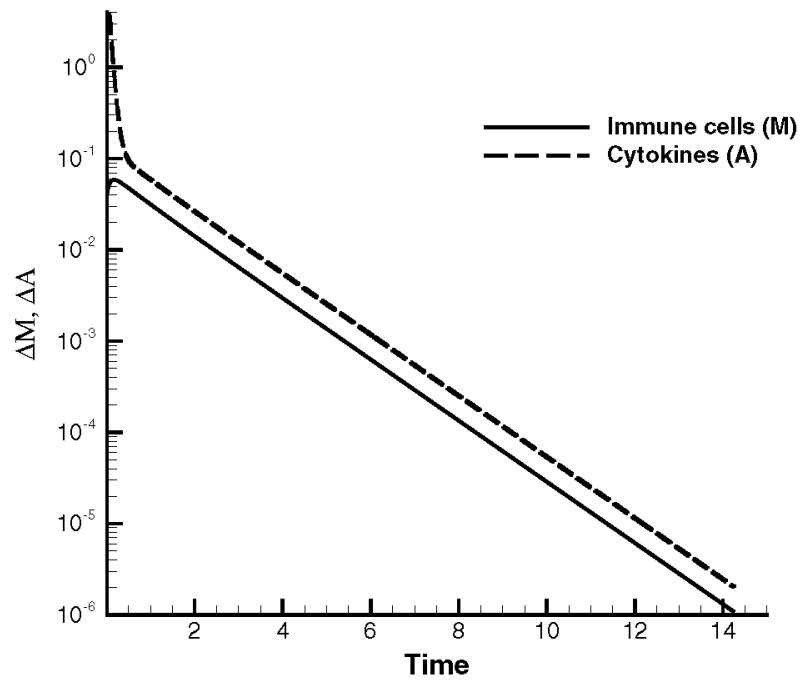

Fig. 13 Convergence of the iterative process leading to the steady state solution computed numerically. We use logarithmic scale for the vertical axis. We measure in the $L_{2}$ norm the distance between the solution for two consecutive times. $\Delta M:=\left\|\mathbf{M}^{n+1}-\mathbf{M}^{n}\right\|_{2} ; \Delta A:=\left\|\mathbf{A}^{n+1}-\mathbf{A}^{n}\right\|_{2}$ where $\mathbf{M}^{n}$ (respectively $\mathbf{A}^{n}$ ) is a vector containing the computed values of the variable $\mathbf{M}$ (respectively $\mathrm{A}$ ) for each control volume at time $t^{n}$

remark that, we do not introduce in the numerical scheme the information about the stationary solutions, it finds them as limit of the evolution solutions (Fig. 13).

\subsection{Algorithm $S^{3}$ (Search of Steady State)}

To compute the steady state solutions of system (1)-(4) we proceed as follows. We compute the $L^{2}$-norm of the difference between two consecutive solutions at times $t_{k}$ and $t_{k+1},\left(M\left(x, t_{k}\right), A\left(x, t_{k}\right)\right)$ and $\left(M\left(x, t_{k+1}\right), A\left(x, t_{k+1}\right)\right)$, respectively. When the difference is small (less than a prescribed small positive tolerance) we assume we are close enough to the $\omega$-limit of the corresponding solution. The algorithm proceeds as follows:

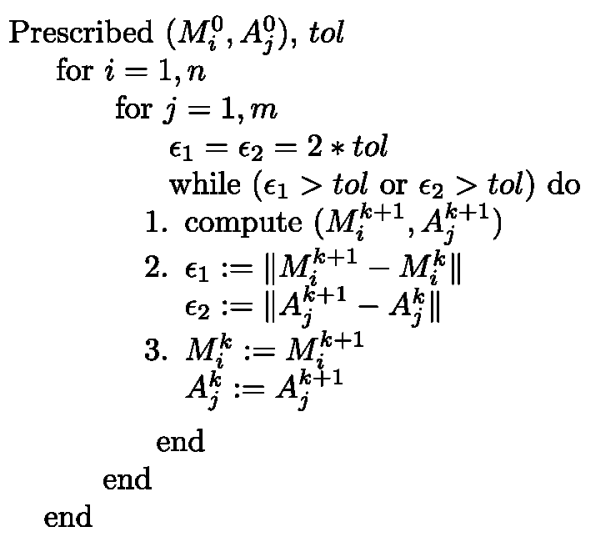


In step $1,\left(M_{i}^{k+1}, A_{j}^{k+1}\right)$ is the approximate solution at time $t_{k+1}$ for initial datum $U_{i j}$, and is obtained using the numerical approach described in Sect. 3. In algorithm $S^{3}$, tol is a fixed positive tolerance that controls the convergence of the iterative process. In this work we have used $t o l=2 \times 10^{-6}$.

\subsection{Qualitative relation to experimental results}

There is experimental evidence that atherosclerosis is an inflammatory process, as assumed in the model of this paper. As mentioned in Poston and Poston (2007), experimental studies suggest that focal atherosclerotic plaques develop through selfperpetuating propagating macrophage recruitment. In vivo experiments indicate that a lesion in the artery usually leads to inflammation that propagates in time. This observation, qualitatively agrees with some of the results obtained in the present paper, as can be seen in Figs. 4, 5, 6, 7, where in most of situations the initial lesion gives rise to a self-propagating process. Moreover, experimental results for mice (Ye et al. 2011) with no pre-existing lesions, that were fed with western-type diet during several weeks, clearly showed the start of a lesion in the artery that developed and increased with time.

Relevant experimental results are also shown in Ikeda and Torii (2005), where the authors experimented by generating atherosclerotic lesions in eleven Japanese macaques. They observed the progress of the lesions under changing feeding conditions. These results are quite relevant to our study. They considered two different scenarios and these are in clear accordance with the two possible situations of our bistable case that corresponds to each set of parameters of Table 2. The first scenario deals with fatty streaks (early lesions of atherosclerosis developed in the aortic wall owing to cholesterol loading) that disappeared when the serum concentration of total cholesterol was reduced. The second scenario involves atheroma, which are advanced lesions that gradually spread to nearby areas even though serum cholesterol was reduced. We see a clear accordance between these experimental information and the results of our bistable case, as displayed in Figs. 4, 5, where small lesions decay with time and finally disappear, whereas large lesions lead to inflammation that progresses with time.

It can be checked in Figs. 5, 6, 7 that the evolution of the inflammation starts progressing slowly but, after a certain time, the inflammation progresses much faster. This agrees with experimental observations, as mentioned in Ibragimov et al. (2005). This is because atherosclerosis is a self-accelerating process.

There are some studies regarding quantitative analysis of cytokines and immune cells produced in the atherosclerosis process. The parameters used in our model are consistent with those proposed in the literature by different authors, see for example Khatib et al. (2009, 2012), Edelstein-Keshet and Spiros (2002), Chow et al. (2005). The time scales are also consistent with the results given, for example, in Chow et al. (2005). In fact, the results displayed in that paper for the concentration of different cytokines are studied in the range $[0,28]$ hours, which agree fairly well with the time scales used in our numerical simulations (see Figs. 4, 5, 6, 7), where time units are hours since the diffusivity coefficients used are given in $\mathrm{cm}^{2} \mathrm{~h}^{-1}$. It is also worth mentioning the recent work in Qi et al. (2012), where the authors make use of experimental techniques for a quantitative study of atherosclerosis progression. See also Nakata et al. 
(2009) where concentration of cytokines in plasma using real-time PCR is measured. Moreover, current bio-medical research makes use of biomedical-imaging techniques to visualisation arterial dimensions, lumen and blood flow. Such techniques facilitate the study of the effects of therapies to treat the plaque (atheroma). We remark, however, that our model is concerned with the simulation of an early stage of the disease.

\section{Analytical study of the atherosclerosis model}

In this section we prove some results regarding some properties of solutions of (1)-(4) and their evolution. The hypothesis of monotonicity of the source terms $f_{1}$ and $f_{2}$, and the well known properties of the Laplacian operator allow us to compare the solution $(M(x, t), A(x, t))$ of (1)-(4) with some other functions to understand its evolution. These properties also guarantee that the upper and lower solutions techniques work for this problem.

A pair of functions $(\bar{M}, \bar{A})$ and $(\underline{M}, \underline{A})$ in $C([0, T] \times[0, L]) \cap C^{1,2}((0, T) \times(0, L))$ are called ordered upper and lower solutions of (1)-(4) if they satisfy $\bar{M} \geq \underline{M}, \bar{A} \geq \underline{A}$ and

$$
\begin{gathered}
\frac{\partial \bar{M}}{\partial t}-d_{1} \frac{\partial^{2} \bar{M}}{\partial x^{2}}-f_{1}(\bar{A})+\lambda_{1} \bar{M} \geq 0 \geq \frac{\partial \underline{M}}{\partial t}-d_{1} \frac{\partial^{2} \underline{M}}{\partial x^{2}}-f_{1}(\underline{A})+\lambda_{1} \underline{M} \\
\frac{\partial \bar{A}}{\partial t}-d_{2} \frac{\partial^{2} \bar{A}}{\partial x^{2}}-f_{2}(\bar{A}) \bar{M}+\lambda_{2} \bar{A} \geq 0 \geq \frac{\partial \underline{A}}{\partial t}-d_{2} \frac{\partial^{2} \underline{A}}{\partial x^{2}}-f_{2}(\underline{A}) \underline{M}+\lambda_{2} \underline{A} \\
\frac{\partial \bar{M}}{\partial x} \geq 0 \geq \frac{\partial \underline{M}}{\partial x}, \quad \frac{\partial \bar{A}}{\partial x} \geq 0 \geq \frac{\partial \underline{A}}{\partial x} \text { on } x=0 \text { and } x=L \\
\bar{M}(x, 0) \geq M_{0}(x) \geq \underline{M}(x, 0), \quad \bar{A}(x, 0) \geq A_{0}(x) \geq \underline{A}(x, 0) .
\end{gathered}
$$

It is well known that the method of upper and lower solution gives the existence of solution of this type of problems [see e.g. Pao (1992)]. Starting from a suitable initial iteration, we construct a sequence $\left(M_{k}, A_{k}\right)$ as follows,

$$
\begin{gathered}
\frac{\partial M_{k}}{\partial t}-d_{1} \frac{\partial^{2} M_{k}}{\partial x^{2}}+\lambda_{1} M_{k}=f_{1}\left(A_{k-1}\right) \\
\frac{\partial A_{k}}{\partial t}-d_{2} \frac{\partial^{2} A_{k}}{\partial x^{2}}+\lambda_{2} A_{k}=f_{2}\left(A_{k-1}\right) M_{k-1} \\
\frac{\partial M_{k}}{\partial x}=0, \quad \frac{\partial A_{k}}{\partial x}=0 \text { on } x=0 \text { and } x=L \\
M_{k}(x, 0)=M_{0}(x), \quad A_{k}(x, 0)=A_{0}(x) .
\end{gathered}
$$

If we choose as initial iteration an upper solution, $(\bar{M}, \bar{A})$, or a lower solution, $(\underline{M}, \underline{A})$, then the sequence $\left(M_{k}, A_{k}\right)$ is monotone and converges to the solution of (1)-(4). By using

$$
\begin{array}{rl}
\alpha_{1} \leq f_{1}(s)=\frac{\alpha_{1}+\beta_{1} s}{1+\frac{s}{\tau_{1}}} \leq \beta_{1} \tau_{1}, & s \geq 0, \\
0 \leq f_{2}(s)=\frac{\alpha_{2} s}{1+\frac{s}{\tau_{2}}} \leq \alpha_{2} \tau_{2} & s \geq 0,
\end{array}
$$


we have that $(\bar{M}, \bar{A})=\left(\frac{\beta_{1} \tau_{1}}{\lambda_{1}}, \frac{\beta_{1} \tau_{1} \alpha_{2} \tau_{2}}{\lambda_{1} \lambda_{2}}\right)$ is an upper solution, and $(\underline{M}, \underline{A})=\left(\frac{\alpha_{1}}{\lambda_{1}}, 0\right)$ is a lower solution and they are ordered. Concerning the uniqueness of the solution, it is a consequence of the locally Lipschitz continuity of the source terms. We have the following bounds for the derivatives of $f_{1}$ and $f_{2}$.

$$
\begin{array}{r}
0<f_{1}^{\prime}(s)=\frac{\beta_{1}-\frac{\alpha_{1}}{\tau_{1}}}{\left(1+\frac{s}{\tau_{1}}\right)^{2}} \leq \beta_{1}-\frac{\alpha_{1}}{\tau_{1}}, \quad s \geq 0, \\
0<f_{2}^{\prime}(s)=\frac{\alpha_{2}}{\left(1+\frac{s}{\tau_{2}}\right)^{2}} \leq \alpha_{2}, \quad s \geq 0 .
\end{array}
$$

Proposition 1 Assume (5), (6), (7) and $\Omega=(0, L)$. For every initial datum $\left(M_{0}(x), A_{0}(x)\right)$ in the rectangle $\left[\frac{\alpha_{1}}{\lambda_{1}}, \frac{\beta_{1} \tau_{1}}{\lambda_{1}}\right] \times\left[0, \frac{\beta_{1} \tau_{1} \alpha_{2} \tau_{2}}{\lambda_{1} \lambda_{2}}\right] \subset L^{\infty}(\Omega) \times L^{\infty}(\Omega)$, the corresponding solution $(M(x, t), A(x, t))$ of (1)-(4) verifies:

$$
\frac{\alpha_{1}}{\lambda_{1}} \leq M(x, t) \leq \frac{\beta_{1} \tau_{1}}{\lambda_{1}} \quad 0 \leq A(x, t) \leq \frac{\beta_{1} \tau_{1} \alpha_{2} \tau_{2}}{\lambda_{1} \lambda_{2}} .
$$

That is, it also lives in the rectangle.

Proof $M_{1}:=\frac{\beta_{1} \tau_{1}}{\lambda_{1}}, A_{1}:=\frac{\beta_{1} \tau_{1} \alpha_{2} \tau_{2}}{\lambda_{1} \lambda_{2}}$. We notice that $\left(M_{1}, A_{1}\right)$ is a solution of the auxiliary problem $\left(P_{1}\right)$

$$
\begin{gathered}
\frac{\partial M}{\partial t}-d_{1} \frac{\partial^{2} M}{\partial x^{2}}+\lambda_{1} M=\beta_{1} \tau_{1} \\
\frac{\partial A}{\partial t}-d_{2} \frac{\partial^{2} A}{\partial x^{2}}+\lambda_{2} A=\alpha_{2} \tau_{2} M_{1} \\
\frac{\partial M}{\partial x}=0, \quad \frac{\partial A}{\partial x}=0 \text { on } x=0 \text { and } x=L \\
M(x, 0)=M_{1}, \quad A(x, 0)=A_{1} .
\end{gathered}
$$

Now, we take the difference between (1) and (53) and multiply by $\left(M-M_{1}\right)^{+}$. We recall that $u^{+}=\max \{0, u\}$. By integrating on $\Omega$, it is verified that

$$
\begin{array}{r}
\frac{d}{d t} \int_{\Omega}\left|\left(M-M_{1}\right)^{+}\right|^{2}+d_{1} \int_{\Omega}\left|\nabla\left(M-M_{1}\right)^{+}\right|^{2}+\lambda_{1} \int_{\Omega}\left|\left(M-M_{1}\right)^{+}\right|^{2} \\
=\int_{\Omega}\left(f_{1}(A)-\beta_{1} \tau_{1}\right)\left(M-M_{1}\right)^{+} \leq 0 .
\end{array}
$$

Then $\frac{d}{d t} \int_{\Omega}\left|\left(M-M_{1}\right)^{+}\right|^{2} \leq 0$. So, we integrate in $(0, t)$ and obtain

$$
\int_{\Omega}\left|\left(M(x, t)-M_{1}\right)^{+}\right|^{2} \leq \int_{\Omega}\left|\left(M_{0}(x)-M_{1}\right)^{+}\right|^{2} .
$$

Since the right hand side is zero then $\left(M-M_{1}\right)^{+}=0$ a.e. $x \in \Omega$ and by the continuity of the solutions, $M(x, t) \leq M_{1}$ for all $(x, t) \in \Omega \times(0,+\infty)$. 
The second equation gives

$$
\begin{aligned}
& \frac{d}{d t} \int_{\Omega}\left|\left(A-A_{1}\right)^{+}\right|^{2}+d_{2} \int_{\Omega}\left|\nabla\left(A-A_{1}\right)^{+}\right|^{2}+\lambda_{2} \int_{\Omega}\left|\left(A-A_{1}\right)^{+}\right|^{2} \\
& =\int_{\Omega}\left(f_{2}(A) M-\alpha_{2} \tau_{2} M_{1}\right)\left(A-A_{1}\right)^{+} \\
& =\int_{\Omega}\left(f_{2}(A)-\alpha_{2} \tau_{2}\right) M\left(A-A_{1}\right)^{+}+\int_{\Omega} \alpha_{2} \tau_{2}\left(M-M_{1}\right)\left(A-A_{1}\right)^{+} \leq 0 .
\end{aligned}
$$

Then, $\frac{d}{d t} \int_{\Omega}\left|\left(A-A_{1}\right)^{+}\right|^{2} \leq 0,\left(A-A_{1}\right)^{+}=0$ a.e. $x \in \Omega$ and $A(x, t) \leq A_{1}$ for $(x, t) \in \Omega \times(0,+\infty)$. Analogously, we obtain the lower bound. By defining $M_{2}:=\frac{\alpha_{1}}{\lambda_{1}}, A_{2}:=0$, we arrive to $\left\|\left(M_{2}-M\right)^{+}\right\|_{L^{2}(\Omega)}=0,\left\|\left(A_{2}-A\right)^{+}\right\|_{L^{2}(\Omega)}=0$. Then $M_{2} \leq M(x, t)$ and $A_{2} \leq A(x, t)$. In fact, $\left(M_{1}, A_{1}\right)$ is an upper solution and $\left(M_{2}, A_{2}\right)$ is an lower solution of the problem.

In fact, we can identify this rectangle of bounded functions with a region in the phase space in a suitable functional space (e.g. $L^{2}(\Omega) \times L^{2}(\Omega)$ or $L^{\infty}(\Omega) \times L^{\infty}(\Omega)$ ). Every initial point in the rectangle gives a trajectory in the rectangle.

We also have that every stationary solution of problem (1)-(4) also lives in the rectangle defined in proposition 1.

Proposition 2 Every stationary solution $(M(x), A(x))$ verifies:

$$
\frac{\alpha_{1}}{\lambda_{1}} \leq M(x) \leq \frac{\beta_{1} \tau_{1}}{\lambda_{1}} \quad 0 \leq A(x) \leq \frac{\beta_{1} \tau_{1} \alpha_{2} \tau_{2}}{\lambda_{1} \lambda_{2}} .
$$

Proof Let $(M(x), A(x))$ be a solution of (11)-(13). Now, we follow the steps in the proof of Proposition 1 to obtain

$$
\begin{aligned}
& d_{1} \int_{\Omega}\left|\nabla\left(M-M_{1}\right)^{+}\right|^{2}+\lambda_{1} \int_{\Omega}\left|\left(M-M_{1}\right)^{+}\right|^{2}=\int_{\Omega}\left(f_{1}(A)-\beta_{1} \tau_{1}\right)\left(M-M_{1}\right)^{+} \leq 0 \\
& d_{2} \int_{\Omega}\left|\nabla\left(A-A_{1}\right)^{+}\right|^{2}+\lambda_{2} \int_{\Omega}\left|\left(A-A_{1}\right)^{+}\right|^{2}=\int_{\Omega}\left(f_{2}(A) M-\alpha_{2} \tau_{2} M_{1}\right)\left(A-A_{1}\right)^{+} \leq 0 .
\end{aligned}
$$

Then, $\left(M-M_{1}\right)^{+}=0,\left(A-A_{1}\right)^{+}=0$ for $x \in \Omega$ and $M(x) \leq M_{1}, A(x) \leq A_{1}$. Analogously, we obtain $M_{2} \leq M(x), A_{2} \leq A(x)$.

Regarding the dynamics of the model, we have studied the $\omega$-limit of different initial data in the last section. Inspired by the numerical results, we prove the following

Proposition 3 Let $\left(M_{\infty}(x), A_{\infty}(x)\right)$ be a solution of the stationary problem (11)(13) and $(M(x, t), A(x, t))$ a solution of the parabolic problem (1)-(4) with initial datum $M(x, 0)=M^{0}(x), A(x, 0)=A^{0}(x)$ verifying

$$
M^{0}(x) \leq M_{\infty}(x), \quad A^{0}(x) \leq A_{\infty}(x) .
$$


Then

$$
M(x, t) \leq M_{\infty}(x), \quad A(x, t) \leq A_{\infty}(x)
$$

Proof Proceed as in Proposition 1 and get the inequality

$$
\begin{aligned}
& \frac{d}{d t} \int_{\Omega}\left|\left(M(x, t)-M_{\infty}(x)\right)^{+}\right|^{2} d x+\frac{d}{d t} \int_{\Omega}\left|\left(A(x, t)-A_{\infty}(x)\right)^{+}\right|^{2} d x \\
& \leq K \int_{\Omega}\left|\left(M(x, t)-M_{\infty}(x)\right)^{+}\right|^{2} d x+K \int_{\Omega}\left|\left(A(x, t)-A_{\infty}(x)\right)^{+}\right|^{2} d x
\end{aligned}
$$

where $K$ is a positive constant depending on the parameters of the problem and the bounds of $f_{1}^{\prime}$ and $f_{2}^{\prime}$. The idea of the proof is based on the following inequality which is obtained by applying Gronwall inequality to (60).

$$
\begin{aligned}
& \left\|\left(M(\cdot, t)-M_{\infty}\right)^{+}\right\|_{L^{2}(\Omega)}^{2}+\left\|\left(A(\cdot, t)-A_{\infty}\right)^{+}\right\|_{L^{2}(\Omega)}^{2} \\
& \quad \leq\left(\left\|\left(M(\cdot, 0)-M_{\infty}\right)^{+}\right\|_{L^{2}(\Omega)}^{2}+\left\|\left(A(\cdot, 0)-A_{\infty}\right)^{+}\right\|_{L^{2}(\Omega)}^{2}\right) e^{K t} .
\end{aligned}
$$

Now it is easy to see that since the right hand side is zero then the left hand side is zero and we obtain the conclusion of Proposition 3.

Remark 1 We notice that Proposition 3 could be useful in the bistable case when we consider $\left(M_{\infty}, A_{\infty}\right)$ equal to the unstable stationary solution. In that case, for every initial datum verifying

$$
M^{0}(x) \leq M_{\infty}(x)-\epsilon_{1} \quad A^{0}(x) \leq A_{\infty}(x)-\epsilon_{2}
$$

the $\omega$-limit is $\left(\frac{\alpha_{1}}{\lambda_{1}}, 0\right)$.

Proposition 4 Let $(M(x, t), A(x, t))$ and $(\hat{M}(x, t), \hat{A}(x, t))$ be the solutions of (1)(4) with initial data $\left(M_{0}(x), A_{0}(x)\right)$ and $\left(\hat{M}_{0}(x), \hat{A}_{0}(x)\right)$. If $M_{0}(x) \leq \hat{M}_{0}(x)$ and $A_{0}(x) \leq \hat{A}_{0}(x)$ a.e. $x \in \Omega$ then $M(x, t) \leq \hat{M}(x, t)$ and $A(x, t) \leq \hat{A}(x, t)$ a.e. $(x, t) \in \Omega \times(0,+\infty)$.

The proof is a consequence of the inequality

$$
\begin{aligned}
& \left\|(M(\cdot, t)-\hat{M}(\cdot, t))^{+}\right\|_{L^{2}(\Omega)}^{2}+\left\|(A(\cdot, t)-\hat{A}(\cdot, t))^{+}\right\|_{L^{2}(\Omega)}^{2} \\
& \quad \leq\left(\left\|\left(M_{0}-\hat{M}_{0}\right)^{+}\right\|_{L^{2}(\Omega)}^{2}+\left\|\left(A_{0}-\hat{A}_{0}\right)^{+}\right\|_{L^{2}(\Omega)}^{2}\right) e^{\hat{K} t}
\end{aligned}
$$

where $\hat{K}$ is a positive constant.

A consequence of Proposition 4 is that if an initial datum is lower bounded and upper bounded by functions which are in the basin of attraction of the same stationary steady state then the initial datum is also in that basin of attraction. 


\section{Conclusion and further studies}

We have studied numerically and analytically a reaction-diffusion type model for atherosclerosis proposed in the literature. We have first developed a high-order nonlinear numerical method to reliably solve the equations numerically. We have then used the numerical method to study the behaviour of time dependent solutions and steady state limits. Computationally, we have classified the initial data predicting the evolution to the disease steady state or the healthy state. This has involved a very intensive computational exercise. For each of the three examples we have solved the complete diffusion-reaction system to steady state 21,504 times. To find the steady state limit we need a large number of time steps, depending on the particular case. To give the reader an idea, for our Example 1, for one particular initial data we employed 1,400 time steps, for $t o l=2 \times 10^{-6}$.

Some of the numerical findings have then been proved rigorously through an analytical study of the system, thus connecting numerics and analysis. We have identified qualitative properties related with the evolution of the density of the immune cells and cytokines with time.

Some of the numerical results reported in the present paper have been qualitatively related to experimental data. In particular, in Ikeda and Torii (2005) the authors experimented by generating atherosclerotic lesions in eleven Japanese macaques and observed the progress of the lesions under changing feeding conditions. The results reported there agree, qualitatively, with the results of our bistable case, where early lesions (fatty streaks) progress towards complete healing whereas large lesions (atheroma) progress towards permanent illness.

In order to better understand atherosclerosis as an inflammatory disease, it would be desirable in the future to include the thickness of the intima of the artery in the model and to study the possible classification of the initial data for a more complete mathematical model.

In Sect. 4.5 we discussed the relation of our simulations to experimental results, from a qualitative point of view, mentioning range of parameters and time scales. It would be highly desirable to carry out a quantitative comparison with experiments, which would require a close collaboration with experimentalists in the field. One way to proceed would be to undertake experiments in vitro, focused on measurement of physical parameters, and also experiments in vivo to measure cytokines and immune cells. This would allow the study of realistic problems and to obtain realistic values of the parameters by solving inverse problems. We believe that with these ad hoc experiments a quantitative assessment could be reliable.

Acknowledgments A.H. thanks Fundación Caja Madrid (Spain) for its financial support by a grant under the programme Becas de movilidad para profesores de las universidades públicas de Madrid. The research of L.T. was partially supported by the research projects MTM2008-06208 and Modelización, Análisis y Control de EDPs, DGISGPI, Ref: MTM2011-26119 (Spain).

\section{References}

Balsara DS, Shu Ch-W (2000) Monotonicity preserving weighted essentially non-oscillatory schemes with increasingly order of accuracy. J Comp Phys 160:405-452 
Calvez V, Houot JG, Meunier N, Raoult A, Rusnakova G (2010) Mathematical and numerical modeling of early atherosclerotic lesions. ESAIM: Proceedings 30:1-14

Cantrell RS, Cosner C (2003) Spatial ecology via reaction-diffusion equations. Wiley series in Mathematical and computational Biology. John Wiley \& Sons, Ltd., Chichester

Cobbold CA, Sherrat JA, Maxwell SRJ (2002) Lipoprotein oxidation and its significance for atherosclerosis: a mathematical approach. Bull Math Biol 64:65-95

Chow CC, Clermont G, Kumar R, Lagoa C, Tawadrous Z, Gallo D, Betten B, Bartels J, Constantine G, Fink MP, Billiar TR, Vodovotz Y (2005) The acute inflammatory response in diverse shock states. Shock 24(1):74-84

Dumbser M, Enaux C, Toro EF (2008) Finite volume schemes of very high order of accuracy for stiff hyperbolic balance laws. J Com Phys 227:3971-4001

Dumbser M (2010) Arbitrary high order PNPM schemes on unstructured meshes for the compressible Navier-stokes equations. Comput Fluids 39(1):60-76

Edelstein-Keshet L, Spiros A (2002) Exploring the formation of Alzheimer's disease senile plaques in silico. J Theor Biol 216(3):301-326

El Khatib N, Genieys S, Volpert V (2007) Atherosclerosis initiation modeled as an inflammatory disease. Math Model Nat Phenom 2(2):126-141

El Khatib N, Genieys S, Kazmierczak B, Volpert V (2009) Mathematical modelling of atherosclerosis as an inflammatory disease. Phil Trans R Soc 367:4877-4886

El Khatib N, Genieys S, Kazmierczak B, Volpert V (2012) Reaction diffusion model of atherosclerosis development. J Math Biol 65(2):349-374. doi:10.1007/s00285-011-0461-1

Gassner G, Lorcher F, Munz CD (2007) A contribution to the construction of diffusion fluxes for finite volume and discontinuous Galerkin schemes. J Comput Phys 224(2):1049-1063. doi:10.1016/j.jcp. 2006.11.004

Godunov SK (1959) A finite difference method for the computation of discontinuous solutions of the equations of fluid dynamics. Mat Sbornik 47:357-393

Herald MC (2010) General model of inflammation. Bull Math Biol 72:765-779. doi:10.1007/ s11538-009-9468-9

Hidalgo A, Dumbser M (2011) ADER schemes for nonlinear systems of stiff advection-diffusion-reaction equations. J Sci Comput 48(1-3):173-189. doi:10. 1007/s10915-010-9426-6

Hidalgo A, Tello L (2011) A finite volume scheme for simulating the coupling between deep ocean and an atmospheric energy balance model. Modern Mathematical Tools and Techniques capturing complexity. Springer, Berlin, pp 239-255

Ibragimov AI, McNeal CJ, Ritter LR, Walton JR (2005) A mathematical model of atherogenesis as an inflammatory response. Math Med Biol 22(4):305-333

Ikeda N, Torii R (2005) When does atherosclerosis become irreversible? Chronological change from an early to an advanced atherosclerotic lesion observed by angioscopy. Angiology 56(4):361-370

Jiang GS, Shu Ch-W (1996) Efficient implementation of weighted ENO schemes. J Comp Phys 126:202_ 228

Levick JR (2010) An introduction to Cardiovascular Physiology. Fifth edition 2010 published in Great Britain by Hodder Arnold, a member of the Hodder Education, an Hachette UK company, London.

Li ZY, Howarth SPS, Tang T, Gillard JH (2006) How critical is fibrous cap thickness to carotid plaque stability? A flow-plaque interaction model. Stroke 37:1195-1199. doi:10.1161/01.STR.0000217331. $61083.3 \mathrm{~b}$

Libby P (2002) Inflammation in atherosclerosis. Nature 420:19-26

Murray JD (2002) Mathematical biology I: an introduction. Springer, Berlin

Nakata M, Itou T, Sakai T (2009) Quantitative analysis of inflammatory cytokines expression in peripheral blood mononuclear cells of the ferret (Mustela putorius furo) using real-time PCR. Vet Immunol Immunopathol 15:130(1-2):88-91 doi:10.1016/j.vetimm.2008.12.009

Ougrinovskaia A, Thompson RS, Myerscough MR (2010) An ODE model of early stages of atherosclerosis: mechanisms of the inflammatory response. Bull Math Biol 72:1534-1561. doi:10.1007/ s11538-010-9509-4

Pao CV (1992) Nonlinear parabolic and elliptic equations. Plenum Press, New York

Poston RN, Poston RM (2007) Typical atherosclerotic plaque morphology. Math Model Nat Phenom 2(02):142-149 
Qi Y, Yafeng L, Ahmed BW et al (2012) Temporal and quantitative analysis of atherosclerotic lesions in diet-induced hypercholesterolemic rabbits. J Biomed Biotech 2012 (Article ID 506159). doi:10.1155/ 2012/506159

Ross R, Glomset JA (1973) Atherosclerosis and the arterial smooth muscle cell. Science 180:1332-1339

Ross R (1993) The pathogenesis of atherosclerosis: a perspective for 1990s. Nature 362:801-809

Ross R (1999) Atherosclerosis: an inflammatory disease. N Engl J Med 340(2):115-126

Schachter M (1997) The pathogenesis of atherosclerosis. Int J Cardiol 62(Suppl. 2):S3_S7

Titarev VA, Toro EF (2004) Finite-volume WENO schemes for three-dimensional conservation laws. J Comp Phys 201:238-260

Titarev VA, Toro EF (2005) ADER schemes for three-dimensional non-linear hyperbolic systems. J Comp Phys 204(2):715-736

Toro EF, Millington RC, Nejad LAM (2001) Towards very high-order Godunov schemes. In: Toro EF (ed) Godunov methods: theory and applications. Edited Review. Conference in Honour of Godunov SK, vol. 1. Kluwer Academic/Plenum Publishers, New York, pp 897-902

Toro EF (2009) Riemann solvers and numerical methods for fluid dynamics: a practical introduction, 3rd edn. Springer, Berlin

Toro EF, Hidalgo A (2009) ADER finite volume schemes for nonlinear reaction-diffusion equations. Appl Num Math 59(1):73-100

Ye D, Zhao Y, Hildebrand RB, Singaraja R, Hayden MR, Van Berkel TJC, Van Eck M (2011) The dynamics of macrophage infiltration into the arterial wall during atherosclerotic lesion development in low-density lipoprotein receptor knockout mice. Am J Pathol 178(1):413-422 